\title{
Motion Planning with Directional Constraints
}

by

Matin Rohani Larijani

A Thesis submitted to

the Faculty of Graduate Studies and Postdoctoral Affairs

in partial fulfilment of

the requirements for the degree of

Master of Science

in

Computer Science

School of Computer Science

Carleton University

Ottawa, Ontario, Canada

May 2014

Copyright (c)

2014 - Matin Rohani Larijani 


\section{Abstract}

In this thesis motion planing with directional constraint in a polygon $P$ possibly with holes is studied. We have studied this problem from two different viewpoints. We proposed an algorithm which directly computes a path that adheres to the directional constraints. We also provided an algorithm which approximates any piecewise linear path in the interior of $P$ with a piecewise linear path that adheres to the directional constraints. 


\section{Contents}

Abstract $\quad$ ii

Table of Contents $\quad$ iii

1 Introduction 1

1.1 Motivation and Background ................. 1

1.2 Problem statement . . . . . . . . . . . . . . . 2

1.3 Thesis organization . . . . . . . . . . . . . 3

2 Finding $G_{D i r}$-admissible paths in two dimensions 4

2.1 Finding a $G_{D i r^{-}}$admissible path in the plane $\ldots \ldots 6$

2.2 Finding a $G_{D i r^{-}}$admissible path in a triangle $\ldots \ldots . . \ldots 10$

2.3 Finding a $G_{D i r^{-}}$admissible path in a polygon $\ldots \ldots \ldots$

3 Approximating a non-admissible path by a $G_{D i r}$-admissible path 20

3.1 Tubular neighborhoods and sleeves . . . . . . . . . . 21

3.2 Approximating a line segment if $G_{D i r}$ is the complete graph . . . . 27

3.3 Approximating a line segment if $G_{D i r}$ is strongly connected . . . . . . 36

3.4 Approximating a path with a $G_{D i r}$-admissible path . . . . . . 45

4 Future Works $\quad 52$

4.1 Approximation in higher dimensions . . . . . . . . . . . 52 


\section{Chapter 1}

\section{Introduction}

\subsection{Motivation and Background}

Path planning algorithms have been the subject of intense investigation in past the fifty years. These efforts have been motivated mainly by robotics but this type of problems arise in many different disciplines and contexts from decision theory and artificial intelligence to control theory and robotics. The general theme of this category of problems is finding a path of consecutive states between an initial state and a goal state through a state space which has a notion of connectivity either defined by a graph or a topology. The path usually is required to satisfy certain constraints and optimality conditions.

Based on the type of the state space these problems can be categorized into discrete, continuous and hybrid group. In the case of discrete problems, the state space is a discrete space and connectivity and constraints are usually defined using a transition graph; this type of problems usually emerge in the context of decision theory. In the continuous case the state space is modeled as a submanifold of $\mathbb{R}^{n}$ and constraints defined by a set of polynomials. The free space, the set of states which satisfy the constraints, forms a semi-algebraic subset of $\mathbb{R}^{n}$. There are various types of constraints which can be applied to the path itself, for example the constraints imposed by the 
kinematics of the system. This type of constraints often expressed using differential forms that the path should satisfy. The hybrid problems like the one arise in self configuring robots and planning with temporal goals use amalgamation of the two other methods.

Solutions to continuous motion planning problems, which is the subject of the work of this thesis, also can be categorized in two groups, sampling based methods and combinatorial methods. Sampling based methods such as PRM [1], RRT [2], PDST [3] and EST [4] have been widely used in modern robotic systems to solve motion planning problems with a variety of kinematics and differential constraints. This type of solutions are probabilistic in nature and are not guaranteed to provide a solution or even terminate. Combinatorial solutions are mostly based on building a one-dimensional road map using a finite decomposition of the state space. Various solutions to Piano Mover problems are an example of this type of solutions [5], [6]. Combinatorial approach to the solution of motion planning problems with kinematic and differential constraints is not studied as extensively as the sampling based methods. To name a few we can mention [7] in which the decidability of the motion planning problem with differential constraints is studied, [8] in which the complexity of approximating a non-constrained path with a constrained path is studied or [9], [10], [11], [12], [13], and [14] in which path planing with curvature constraint constraints is studied.

\subsection{Problem statement}

In this thesis we will study the path planning problem with directional constraint. We study the problem of path planning in the presence of differential constraint in very basic setting in which only a finite set of directions are allowed and a transition graph $G_{D i r}$ defines how the directions are switched from one to another. Let $P \in \mathbb{R}^{2}$ be a polyhedral environment , $V=\left\{v_{1}, \cdots, v_{n}\right\}$ a discrete set of directions, and $G_{\text {Dir }}$ 
a transition graph which has $V$ as its vertices and defines how directions can change, we study the problem of finding paths between any two point in $P$ which adhere to the directional constraints imposed by the transition graph $G_{\text {Dir }}$. These constraints dictate that in each leg of the path we can only move in a direction from the set of allowed directions $V$ and if we change our direction from $v_{1}$ to $v_{2}$ in $V$ then there should be an edge from $v_{1}$ to $v_{2}$ in $G_{D i r}$. We call such a path a $G_{D i r}$-admissible path.

\subsection{Thesis organization}

In Chapter 2 we define some properties for $G_{D i r}$ and establish a relationship between those properties and the problem of finding an admissible path. In the first section of this chapter we will study the problem of finding a $G_{D i r}$-admissible path between two points in the absence of any obstacle and show that if $G_{D i r}$ is symmetric and spanning then we can find a $G_{D i r}$-admissible path with only one change of direction. We also study the problem of finding a $G_{D i r^{-}}$admissible path in a triangle when the allowed directions are aligned with the $x$ and $y$ axes and $G_{D i r}$ is a complete graph. Using the result from this section we show that the performance of any algorithm that solves the $G_{\text {Dir }}$-admissible path planning problem is sensitive to the start and end points.

In Chapter 3, we study the complexity and performance of the approximation of a piecewise linear path by a $G_{D i r^{-}}$-admissible path. We show that if $G_{D i r}$ is symmetric, spanning and strongly connected then any piecewise linear collision free path can be approximated by a $G_{D i r^{-}}$admissible path. We also show that the performance of the approximation is related to how dense the set of allowed direction is, i.e., the maximum angular difference between two consecutive direction in $V$.

Chapter 4 is dedicated to future works and different generalization of materials discussed in the previous Chapters. 


\section{Chapter 2}

\section{Finding $G_{D i r}$-admissible paths in two dimensions}

In this chapter we first formally define the motion planning problem with directional constraints. We define some properties for $G_{D i r}$ and show that if $G_{\text {Dir }}$ have those properties we can find a $G_{D i r}$-admissible path when the environment is the whole $\mathbb{R}^{2}$. To solve our problem for polygonal environment we focus our attention to a triangle and the simplest spanning and symmetric direction transition graph $G_{\text {Dir }}$, i.e. complete graph over north, south, east and west directions, and show that motion planning with directional constraints can be solved in this setting. Next we generalize this results to any polygonal environment $P$ using triangulation. If $v_{1}$ and $v_{2}$ are two arbitrary linearly independent directions in $\mathbb{R}^{2}$ then one can always find a linear isomorphism $T$ which maps the ordered base $\left(v_{1}, v_{2}\right)$ to $((1,0),(0,1))$. Since any triangulation of $P$ is defines a natural triangulation for $T(P)$, we can generalize this results to any complete $G_{\text {Dir }}$.

Let $P$ be a polygonal environment, i.e., a simple polygon, possibly with holes, and let $n$ be the number of vertices of $P$. Let $V=\left\{v_{1}, \ldots, v_{m}\right\}$ be the set of admissible directions, and let $G_{D i r}$ be the direction transition digraph of $V$. This graph has vertex set $V$ and $\left(v_{i}, v_{j}\right)$ is an edge if we can change direction to $v_{j}$ if $v_{i}$ is the current 
direction.

We define the following properties of $V$ and $G_{D i r}(V)$.

Definition 1. We call $V$ symmetric if for each $v \in V,-v$ also belongs to $V$.

Definition 2. For each $v \in V$ we define $\delta^{+}(v)=\left\{v^{\prime}:\left(v, v^{\prime}\right) \in E_{G_{D i r}}\right\}$.

Definition 3. $G_{D i r}$ is spanning if for each vertex $v_{i}$, and for each $v_{j} \in \delta^{+}\left(v_{i}\right),\left\{v_{i}, v_{j}\right\}$ is spanning $\mathbb{R}^{2}$.

Definition 4. If $V$ is symmetric then we define $G_{D i r}$ to be symmetric if for each $v \in V, v^{\prime} \in \delta^{+}(v)$ if and only if $-v^{\prime} \in \delta^{+}(v)$.

Definition 5. Given a pair $(s, e)$ of points inside $P$, a piecewise linear path $R=$ $\left(x_{1}=s, x_{2}, \cdots, x_{k}=e\right)$ inside $P$ is called $G_{D i r}$-admissible, if for each $1 \leq i<k$, there exists a real number $c_{i}>0$ and an element $v_{i}$ in $V$ such that $x_{i+1}-x_{i}=c_{i} v_{i}$ and $v_{i+1} \in \delta^{+}\left(v_{i}\right) . R$ is called strongly $G_{D i r}$-admissible if it is $G_{D i r^{-a d m i s s i b l e ~}}$ and we have $x_{2}-x_{1}=c_{2} v_{s}$ and $x_{k}-x_{k-1}=c_{k} v_{e}$ for given start and end directions $\left(v_{s}, v_{e}\right)$ in $V$.

It can be easily observed that if $G_{D i r}$ is symmetric then in Definition 5 we can assume $x_{i+1}-x_{i}=c_{i} v_{i}$ for an arbitrary real $c_{i}$.

Lemma 6. Given a pair $(s, e)$ of points inside $P, a G_{D i r}$-admissible path for a symmetric $G_{\text {Dir }}$ is a piecewise linear path $R=\left(x_{1}=s, x_{2}, \cdots, x_{k}=e\right)$ inside $P$ such that for each $1 \leq i<k$, there exists a real number $c_{i}$ and an element $v_{i}$ in $V$ such that $x_{i+1}-x_{i}=c_{i} v_{i}$ and $v_{i+1} \in \delta^{+}\left(v_{i}\right)$.

The problem considered in this work can be expressed as:

Problem 7. Given $V=\left\{v_{1}, \ldots, v_{m}\right\}$, a spanning direction transition graph $G_{\text {Dir }}$ and a polygonal environment $P$. For any two query points $(s, e)$, report a $G_{D i r}$-admissible path inside $P$ if such a path exists, or report that such a path does not exist. 


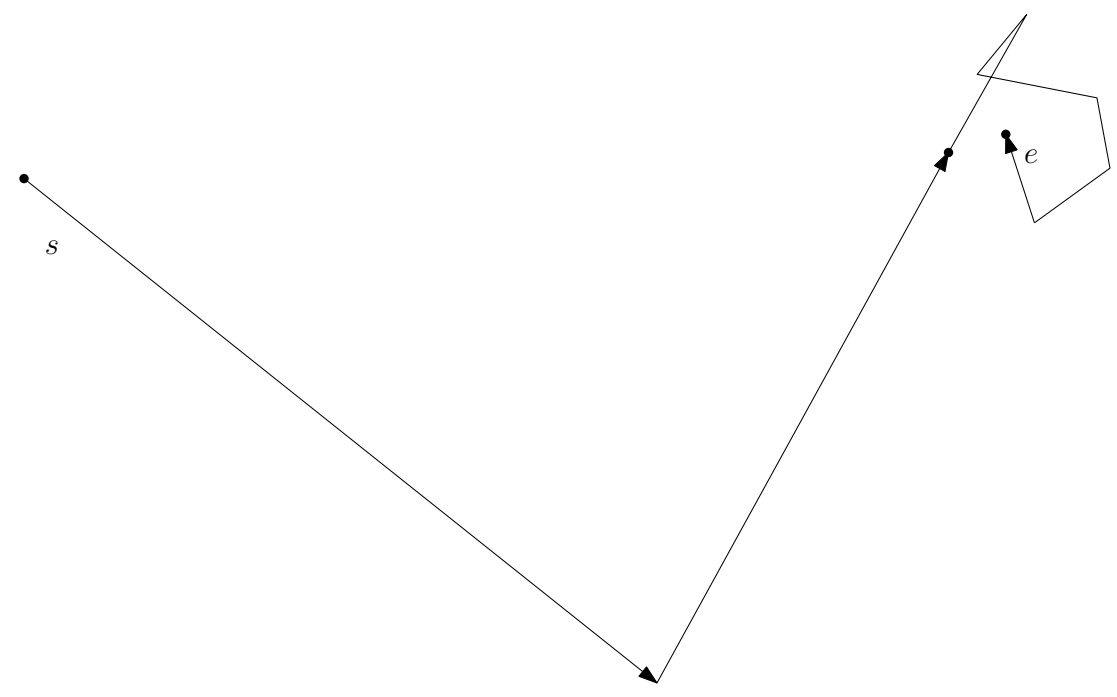

Figure 2.0.1: Sample run of algorithm 1 on $(s, e),\left(v_{s}, v_{e}\right)$.

The strong version of Problem 7 becomes:

Problem 8. Given $V=\left\{v_{1}, \ldots, v_{m}\right\}$, a spanning direction transition graph $G_{\text {Dir }}$ and a polygonal environment $P$. For any two query points $(s, e)$ and start and end directions $\left(v_{s}, v_{e}\right)$ in $V$, report a strongly $G_{\text {Dir }}$ admissible path inside $P$ if such a path exists, or report such a path does not exist.

\subsection{Finding a $G_{D i r}$-admissible path in the plane}

If $P=\mathbb{R}^{2}$ (i.e., there are no constraints on the environment) and $G_{\text {Dir }}$ is both symmetric and spanning and if each edge in $G_{\text {Dir }}$ has unit weight then both the strong and weak versions of the problem can be solved in time that is within a constant factor of finding shortest path in $G_{D i r}$.

For simplicity we will assume that $G_{\text {Dir }}$ is strongly connected. This assumption is not critical because if there is no path between $v_{s}$ and $v_{e}$ then there won't be any $G_{D i r}$ - admissible path between $s$ and $e$ either. We also assume that $v_{s} \neq v_{e}$. This is to avoid finding a cycle that starts and ends at $v_{s}$. The same technique can be used 
to solve the problem when $v_{s}=v_{e}$ by concatenating solutions for input data $(s, c)$ and $\left(v_{s}, v_{c}\right)$, and $(c, e)$ and $\left(v_{c}, v_{s}\right)$, for which $v_{s} \neq v_{c}$.

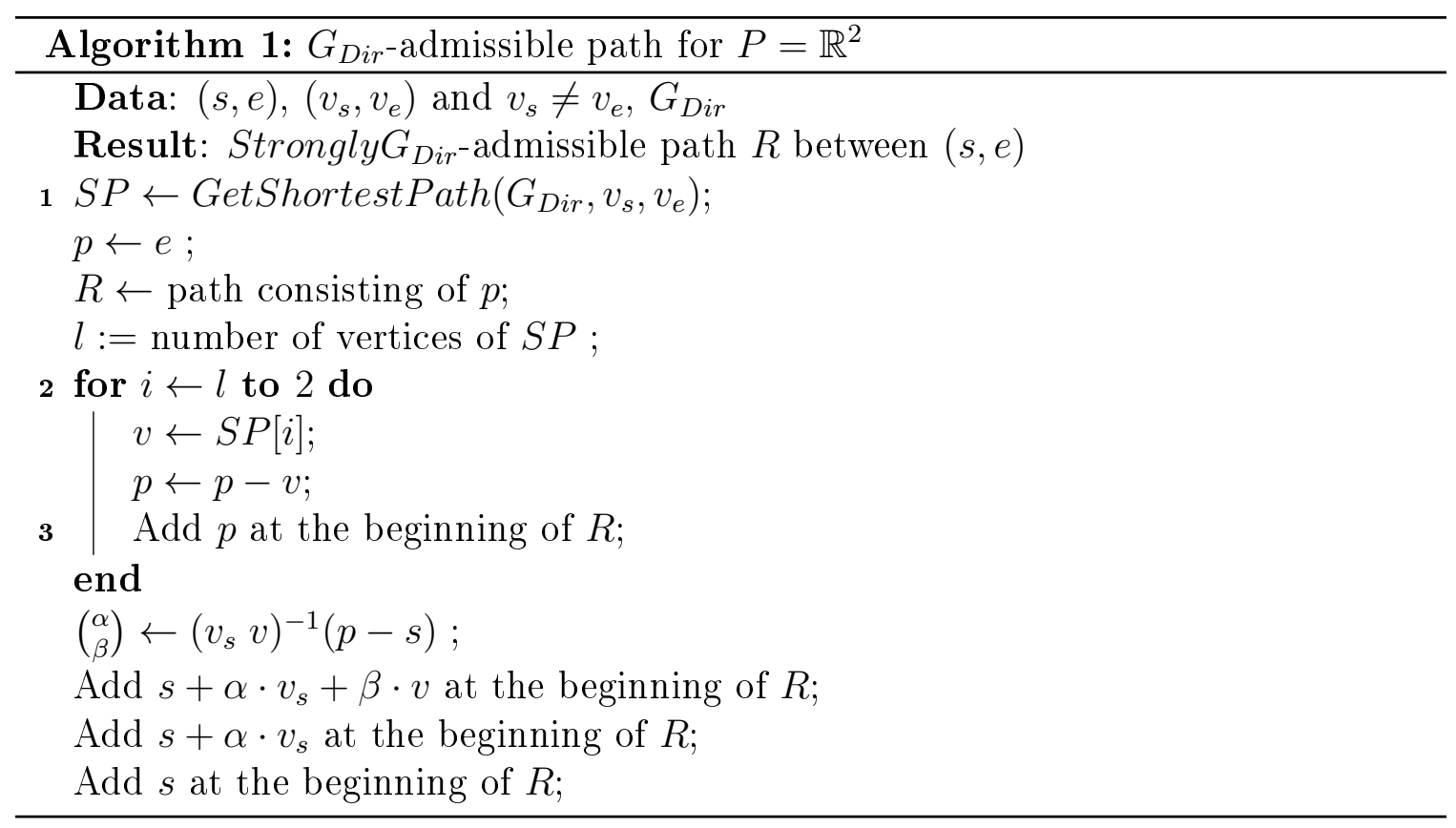

Algorithm 1 provides a solution to Problem 8 with the minimum number of direction changes. Given start and end points $(s, e)$ and distinct start and end directions $\left(v_{s}, v_{e}\right)$, it performs the following steps:

1. Calculate the path $\pi\left(v_{s}, v_{e}\right)$ between $v_{s}$ and $v_{e}$ in $G_{D i r}$ having the minimum number of edges.

2. Starting from $e$ and from the end $v_{e}$ of $\pi\left(v_{s}, v_{e}\right)$. it traverses $\pi\left(v_{s}, v_{e}\right)$ backwards and for each vertex $v_{i}$, it moves a distance of $v_{i}$ in the opposite direction of $v_{i}$.

3. In the last step when it is on the first edge of $\pi\left(v_{s}, v_{e}\right)$, i.e., $\left(v_{s}, v_{1}\right)$, it calculates the distance we should take in direction $v_{1}$ and then $v_{s}$ in order to reach $s$. This can also be achieved by finding the intersection of lines $l\left(p, v_{1}\right)$ and $l\left(s, v_{s}\right)$ which pass through $p$ and $s$ in direction $v_{1}$ and $v_{s}$ respectively. 
Theorem 9. If $P=\mathbb{R}^{2}$ (i.e. there is no constraint on the environment) and $G_{\text {Dir }}$ is both symmetric and spanning then Algorithm 1 solves both the strong and weak versions of the $2 D$ problem within a constant factor of the running time of GetShortestPath. Here, GetShortestPath returns a shortest path between $v_{s}$ and $v_{e}$ in $G_{\text {Dir }}$ according to a constant uniform edge weight (e.g., 1 for all edges). The path generated by Algorithm 1 has the minimum number of links.

Proof: We will show that Algorithm 1 solves the strong case. The weak case can be converted to the strong case by adding the vector $v_{s}$ in $V$ with least angular difference with $\overline{s e}$ (the line segment between $s$ and $e$ ) and $v_{e}$ as a vector in $\delta^{+}\left(v_{s}\right)$ with least angular difference with $v_{s}$.

Let $S P=\left(s p_{1}, \cdots, s p_{l}\right)$ in line 1 be the shortest path between $v_{s}\left(=s p_{1}\right)$ and $v_{e}\left(=s p_{l}\right)$ with length $l$. The for loop starting on line 2 iterates through the last $l-1$ nodes of $S P$ and builds a primary path $R$ between $p$ and $e$. It is immediately observed that $R$ is $G_{D i r}$-admissible by definition.

At line 3 it is guaranteed that $v \in \delta^{+}\left(v_{s}\right)$ and $R$ is a $G_{D i r}$-admissible path between $p$ and $e$. Since $\left(v_{s}, v\right)$ is spanning we have $p-s=\alpha v_{s}+\beta v$ for some real numbers $\alpha$ and $\beta$, so $\left(s, s+\alpha v_{s}, s+\alpha v_{s}+\beta v\right)$ is a $G_{D i r}$-admissible path between $s$ and $p$. Let $\left(\begin{array}{ll}v_{s} & v\end{array}\right)$ be a 2 by 2 matrix with column vectors $v_{s}$ and $v$. Then $\left(v_{s} v\right)\left(\begin{array}{l}1 \\ 0\end{array}\right)=v_{s}$ and $\left(\begin{array}{ll}v_{s} & v\end{array}\right)\left(\begin{array}{l}0 \\ 1\end{array}\right)=v$. Assuming $T=\left(v_{s} v\right)^{-1}$ we have

$$
T(p-s)=\alpha T\left(v_{s}\right)+\beta T(v)=\alpha\left(\begin{array}{l}
1 \\
0
\end{array}\right)+\beta\left(\begin{array}{l}
0 \\
1
\end{array}\right)
$$

So $\alpha$ and $\beta$ can be calculated by matrix formula in the Algorithm 1.

It is clear that the running time of the Algorithm is within a constant factor of the running time of algorithm GetShortestPath. It is also clear that each vertex in $S P$ represent a link in $R$ and as a result the number of vertices of $S P$ is equal to the number of links in $R$. This shows that $R$ is a $G_{D i r^{-}}$admissible path with the minimum 
number of links.

Both the result and complexity of the shortest path function is dependent on the distance function defined on $G_{D i r}$. If unit weight is considered, shortest paths can be computed in $O\left(E_{G_{D i r}}+V_{G_{D i r}}\right)$ using breadth-first search. If different weighting is used Dijkstra's algorithm with Fibonacci heap can be used to achieve $O\left(E_{G_{D i r}}+\right.$ $\left.V_{G_{D i r}} \log \left(V_{d i r}\right)\right)$. Various weights can be used on $G_{D i r}$. For our next result we will define the weight of an edge $\left(v_{i}, v_{j}\right) \in E_{G_{D i r}}$ to be $d\left(0, v_{i}\right)$ where $d$ is any metric on $\mathbb{R}^{2}$.

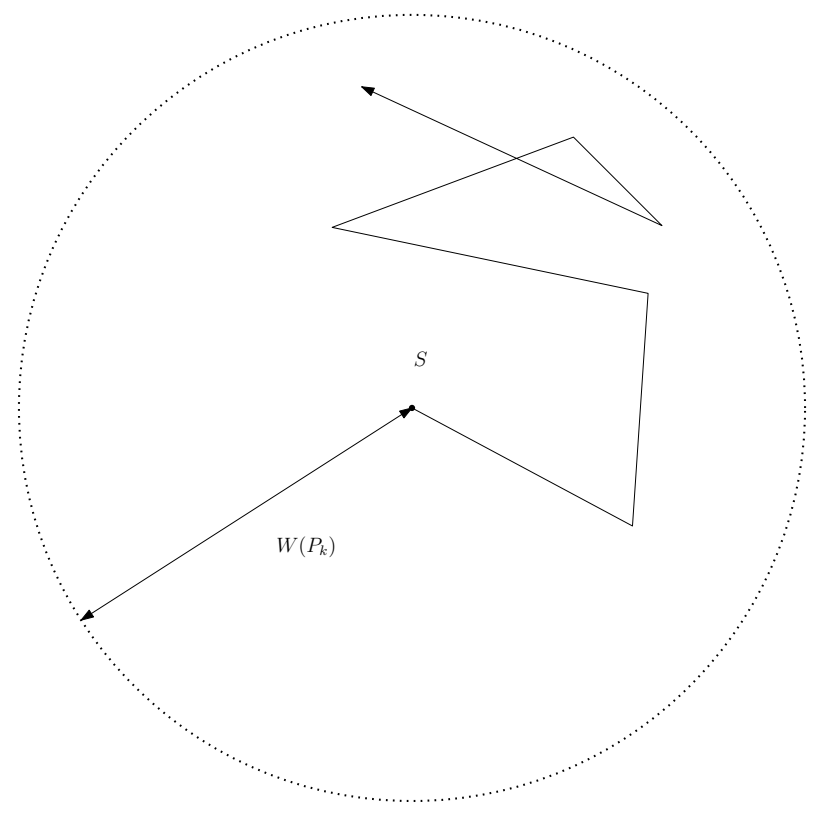

Figure 2.1.1:

Lemma 10. Let $d$ be a given metric on $\mathbb{R}^{2}$ and assume the weight of an edge $\left(v_{i}, v_{j}\right) \in$ $E_{G_{D i r}}$ is $W_{\left(\left(v_{i}, v_{j}\right)\right)}=d\left(0, v_{j}\right)$. Then for any path $P=\left(v, v_{1}, \cdots, v^{\prime}\right)$ between $v$ and $v^{\prime}$ in $G_{\text {Dir }}$ and any given starting point $s \in \mathbb{R}^{n}$, the piecewise linear path $R=\left(r_{0}, \cdots, r_{l}\right)$, in which $r_{0}=s$ and $r_{i}=r_{i-1}+v_{i}$, is contained in the close ball of radius $W(P)=$ $\Sigma_{\left(v_{i}, v_{j}\right) \in P} d\left(0, v_{i}\right)$ centered at $s$. 
Proof: This statement is obvious if $l=1$. Let us assume that this statement is correct for all paths up to length $k$. We will show that it is correct for $k+1$.

If the length of $P$ is $k+1$ then for $P_{k}$, the first $k$ vertices of $P$, the above statement is correct so we can assume that $R_{k}$, the fist $k$ pieces of the associated piecewise linear path $R$, is entirely in a ball of radius $W\left(P_{k}\right)$ as depicted in figure 2.1.1. Now if $x \in \overline{r_{k} r_{k+1}}$ then $d(s, x) \leq d\left(s, r_{k}\right)+d\left(r_{k}, x\right)$ but from the induction hypothesis $\left.d\left(s, r_{k}\right) \leq W\left(P_{k}\right)\right)$ and we also have $d\left(r_{k}, x\right) \leq d\left(r_{k}, r_{k+1}\right)$. So $d(s, x) \leq W(P)$ hence $x \in \overline{B_{d}\left(s, W\left(P_{k}\right)\right)}$.

To avoid complications involved in computing the Euclidean norm due to calculating the square root of sums, the maximum (uniform, supremum) metric $d_{\text {Max }}$ can be used where $d_{M a x}(x, y)=\max _{i}\left|x_{i}-y_{i}\right|$ for $x_{i}$, the $i$-th coordinate of $x$.

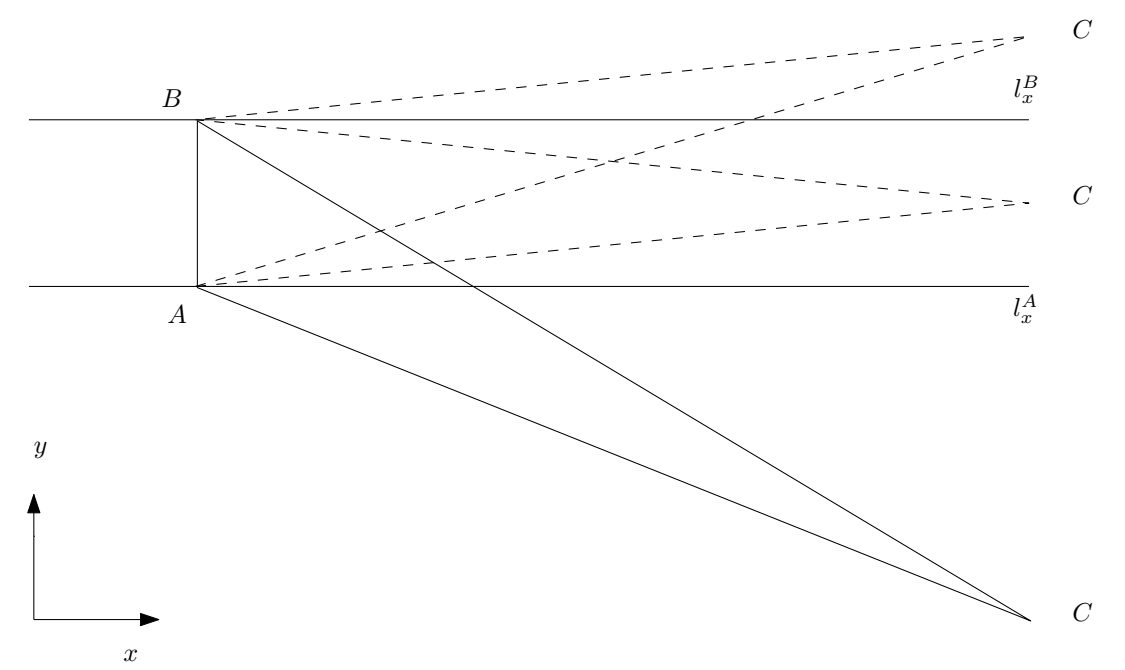

Figure 2.1.2: Position of triangle $A B C$ in relation to $l_{x}^{A}$ and $l_{x}^{B}$

\subsection{Finding a $G_{D i r}$-admissible path in a triangle}

Now we study the problem of finding a $G_{\text {Dir }}$ admissible path in a triangle $A B C$. If $v_{1}$ and $v_{2}$ are two arbitrary linearly independent directions in $\mathbb{R}^{2}$ then one can always find a linear isomorphism $T$ which maps the ordered base $\left(v_{1}, v_{2}\right)$ to $((1,0),(0,1))$. 
Since the image of $A B C$ under $T$ is also a triangle, for simplicity we assume that we are only allowed to move in 4 directions and $G_{d i r}=K_{4}$, the complete digraph of size 4 , and we are only moving in perpendicular directions. Let these 4 directions be denoted by $x=(1,0),-x, y=(0,1)$ and $-y$ to identify them with the normal $\bar{x}$ and $\bar{y}$ axis of the plane. We also assume that the edge $A B$ of the triangle $A B C$ is parallel to $y$ (or $-y$ ). We also denote the $x$ and $y$ components of a point $p \in \mathbb{R}^{2}$ by $x_{p}$ and $y_{p}$. A line passing through a point $p$ in the direction of a vector $v \in \mathbb{R}^{2}$ is denoted by $l_{v}^{p}$. Consider two lines $l_{x}^{A}$ and $l_{x}^{B}$ parallel to direction $x$ and passing through $A$ and $B$ respectively. Two different situations can happen depending on the placement of point $C$.

1. $C$ is between $l_{x}^{A}$ and $l_{x}^{B}$.

2. $C$ is outside $l_{x}^{A}$ and $l_{x}^{B}$.

If $C$ is between $l_{x}^{A}$ and $l_{x}^{B}$ then any pair $\left(s, e^{\prime}\right)$ of points inside $A B C$ can be connected to each other with a $G_{D i r}$-admissible path of length 2. This is illustrated in Figure 2.2.1 for the case when $s$ is to the left of $e$.

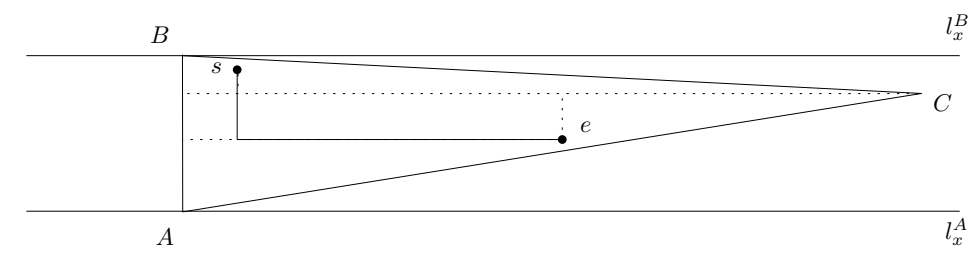

Figure 2.2.1: Finding an admissible path when $C$ is between $l_{x}^{A}$ and $l_{x}^{B}$

Lemma 11. If $A B C$ is a triangle such that the edge $A B$ is parallel to $y$ and vertex $C$ is between $l_{x}^{A}$ and $l_{x}^{B}$, then for any given points $(s, e)$ there exist a $G_{\text {Dir-admissible }}$ 
path of length 2 connecting s to e. Here, $G_{D i r}$ is the complete graph of size 4 over $\{x,-x, y,-y\}$.

Now let us assume that $C$ is outside of $l_{x}^{A}$ and $l_{x}^{B}$. Without loss of generality we can assume that $C$ resides below the line $l_{x}^{A}$ as shown in Figure 2.2.2. Let $p_{-1}=B$ and $p_{0}=A$ and for $k \geq 1, p_{2 k-1}=l_{x}^{p_{2 k-2}} \cap B C$ and $p_{2 k}=l_{y}^{p_{2 k-1}} \cap A C$. This generates an (infinite) triangulation of $A B C$ with triangles $t_{i}=p_{i-1} p_{i} p_{i+1}$.

We have $\lambda=\|A B\|=\left|y_{A}-y_{B}\right|$ and also $\tan (\beta)=\|O A\| /\|O C\|=\left|A_{x}-C_{x}\right| / \mid y_{A}-$ $y_{C} \mid$ and $\cot (\alpha)=\|O C\| /\|O B\|=\left|x_{A}-x_{C}\right| /\left|y_{A}-y_{C}\right|$. Using the similarity of triangles $t_{2 k}$ with $B O C$ and $t_{2 k+1}$ with $A O C$ one can calculate the $\bar{x}$ and $\bar{y}$ coordinates of each $p_{i}$.

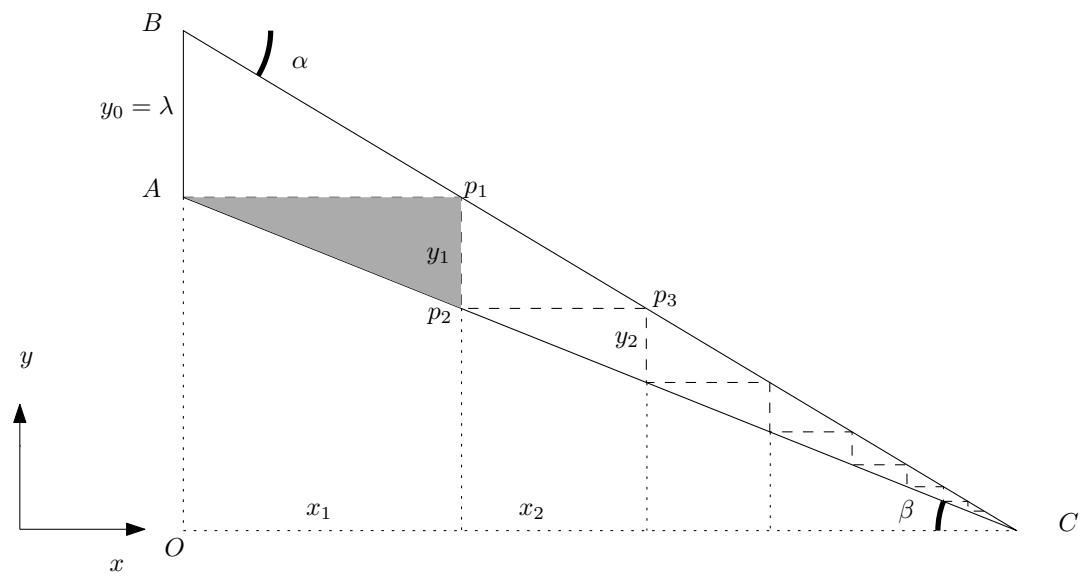

Figure 2.2.2: Partitioning a triangle

We have: 


$$
\begin{aligned}
\left|y_{p_{-1}}-y_{p_{0}}\right| & =\lambda \\
\left|x_{p_{0}}-x_{p_{1}}\right| & =\lambda \cot (\alpha) \\
\left|y_{p_{1}}-y_{p_{2}}\right| & =\lambda \cot (\alpha) \tan (\beta) \\
\left|y_{p_{2 k-1}}-y_{p_{2 k}}\right| & =\lambda(\cot (\alpha) \tan (\beta))^{k} \\
\left|x_{p_{2 k}}-x_{p_{2 k+1}}\right| & =\lambda(\cot (\alpha) \tan (\beta))^{k} \cot (\alpha)
\end{aligned}
$$

Considering the above formulation the $\bar{y}$ coordinate of each point $p_{i}$ can be calculated as a geometric series. We have $y_{p_{2 k}}=y_{B}-\lambda \Sigma_{k=0}^{k}(\cot (\alpha) \tan (\beta))^{k}$ or

$$
y_{p_{2 k}}=y_{B}-\lambda \frac{1-(\cot (\alpha) \tan (\beta))^{k+1}}{1-\cot (\alpha) \tan (\beta)}
$$

For any $t_{2 k}$ and $k \geq 0$, it can be observed that $p_{2 k-1} p_{2 k}$ is parallel to $\bar{y}$ and $p_{2 k+1}$ is between $l_{x}^{p_{2 k-1}}$ and $l_{x}^{p_{2 k}}$. For $t_{2 k+1}$ and $k \geq 0, p_{2 k+1} p_{2 k+2}$ is parallel to $y$ and $p_{2 k}$ is between $l_{x}^{p_{2 k+2}}$ and $l_{x}^{p_{2 k+1}}$. This means that, for any $i \geq 0$, if $(s, e)$ are points inside $t_{i}$ then they can be connected to each other based on Lemma 11.

Now let us assume that $(s, e)$ are given inside $A B C$ the following algorithm calculates an admissible path between $s$ and $e$.

\section{Algorithm 2.}

Input: triangle $A B C$ and start and end point $(s, e)$

Output: $G_{\text {Dir- }}$ admissible path between s and e.

1. Select closest of $s$ and $e$ to the edge $A B$ and denote it by $s_{0}$.

2. Shoot a ray $R\left(s_{0}, x\right)$ from $s_{0}$ parallel to $x$ and away from $A B$ until it hits $B C$ at $s_{1}$. If $l_{y}^{e}$, the line passing through e and parallel to $y$, intersects with $R\left(s_{0}, x\right)$ inside $A B C$ then report the admissible path and stop otherwise continue to the 
next step.

3. Shoot a ray $R\left(s_{1}, y\right)$ from $s_{1}$ vertically down until it hits $A C$ at $s_{2}$. If $l_{x}^{e}$, the line passing through e and parallel to $x$, intersects with $R\left(s_{1}, y\right)$ inside $A B C$ then report the admissible path and stop otherwise set $s_{0}$ to $s_{2}$ and start from step 2.

Figure 2.2.3 shows a run of the above algorithm.

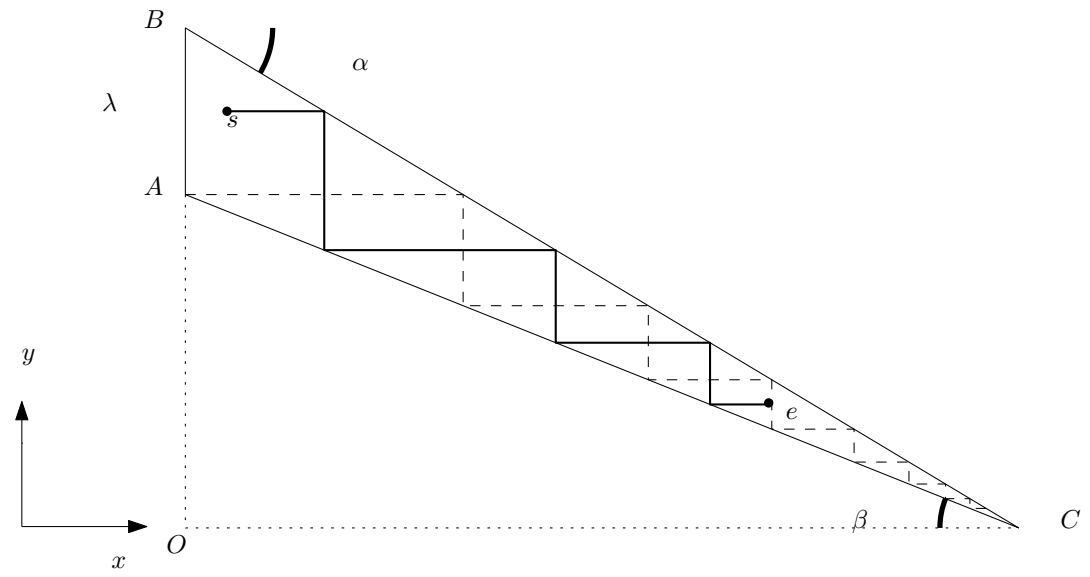

Figure 2.2.3: Finding a $G_{D i r}$-admissible path in a triangle

Let integers $l \leq m$ be selected such that $s \in t_{l}$ and $e \in t_{m}$. It is easy to show that algorithm 2 generates a $G_{D i r}$-admissible path. It is also easy to observe that if the path generated by algorithm 2 enters $t_{m}$ it finishes. It can also be observed that any $G_{D i r}$ - admissible path between $(s, e)$ intersects all triangles $t_{q}$ where $l \leq q \leq m$. This shows that algorithm 2 finishes in finite time. As a matter of fact for any point $p$ in $A B C$ we can find the index $i_{p}$ of the triangle $t_{i_{p}}$ to which $p$ belongs. To do that first we find the index $k$ for which we have $y_{p_{2 k}} \geq y_{p} \geq y_{p_{2(k+1)}}$ or

$$
y_{B}-\lambda \frac{1-(\cot (\alpha) \tan (\beta))^{k+1}}{1-\cot (\alpha) \tan (\beta)} \geq y_{p} \geq y_{B}-\lambda \frac{1-(\cot (\alpha) \tan (\beta))^{k+2}}{1-\cot (\alpha) \tan (\beta)}
$$

Setting $\cot (\alpha) \tan (\beta)=O A / O B=y_{A} / y_{B}$ we have

$$
\left.\left(\frac{O A}{O B}\right)^{k+1} \leq 1-\frac{y_{B}-y_{p}}{y_{B}} \leq \frac{O A}{O B}\right)^{k+2}
$$


Since $\log$ is increasing we have $k=\left\lfloor\log _{O A / O B}\left(1-\frac{y_{B}-y_{p}}{y_{B}}\right)\right\rfloor-1$. Thus, $p$ belongs to $t_{2 k}$ or $t_{2 k+1}$ (i.e. $2 k \leq i_{p} \leq 2 k+1$ ). By examining the value of $x_{p}$ against $x_{p_{2 k}}$ and $x_{p_{2 k+1}}$ the index $i_{p}$ can be exactly determined.

Based on the above discussion we require

$$
|m-l|=\left|\left\lfloor\log _{O A / O B}\left(1-\frac{y_{B}-y_{s}}{y_{B}}\right)\right\rfloor-\left\lfloor\log _{O A / O B}\left(1-\frac{y_{B}-y_{e}}{y_{B}}\right)\right\rfloor\right| \pm 2
$$

steps to finish algorithm 2. Figure 2.2.3 shows a run of algorithm 2.

Lemma 12. If $A B C$ is a triangle such that the edge $A B$ of the triangle $A B C$ is parallel to $y$ and vertex $C$ is outside $l_{x}^{A}$ and $l_{x}^{B}$, parallel lines passing through $A$ and $B$ and parallel to $x$, then for any given points $(s, e)$ there exist a $G_{\text {Dir }}$ - admissible path with

$$
\mid\left\lfloor\log _{O A / O B}\left(1-\frac{y_{B}-y_{s}}{y_{B}}\right)\right\rfloor-\left\lfloor\log _{O A / O B}\left(1-\frac{y_{B}-y_{e}}{y_{B}}\right)\right\rfloor \pm 2
$$

links connecting s to e. Here, $G_{\text {Dir }}$ is complete graph of size 4 over $\{x,-x, y,-y\}$.

Now let $A B C$ be an arbitrary triangle in $\mathbb{R}^{2}$. We can partition $A B C$ into two triangles each sharing a common edge parallel to $y$. This can be done by sorting the points based on their $\bar{x}$ coordinates and picking the middle point. Assuming $C$ has such a property then the line $l_{x}^{C}$ meets the line segment $A B$ at $C^{\prime} . A C C^{\prime}$ and $B C C^{\prime}$ partition $A B C$ into two triangles that meet the requirements of Lemmas 11 and 12 since $C C^{\prime}$ is parallel to $y$. As a result algorithm 2 can be extended to general triangles as follows.

\section{Algorithm 3.}

Input: triangle $A B C$ and start and end point $(s, e)$

output: $G_{D i r^{-}}$admissible path between s and e. 
1. Order the vertices of $A B C$ based on their $x$ coordinates, select the middle vertex (assume $C$ is the middle one) and shoot a line from $C$ which meet $A B$ at $C^{\prime}$. Keep the resulting triangles $A C C^{\prime}$ and $B C C^{\prime}$

2. Find $T_{1}$ the triangle that contains $s$, find $T_{2}$ the triangle which contains $e$.

3. If $T_{1}=T_{2}$ then run algorithm 2 for $T_{1}$ and $(s, e)$.

4. If $T_{1} \neq T_{2}$ then from $\left\{C, C^{\prime}\right\}$ select the one which has the closest $y$ coordinate to e (assume $C^{\prime}$ does). Run algorithm 2 for $T_{1}$ and $\left(s, C^{\prime}\right)$, run algorithm 2 for $T_{2}$ and $\left(C^{\prime}, e\right)$ and concatenate the two resulting paths.

Figure 2.2.4 illustrates how algorithm 3 is applied for a case where $T_{1} \neq T_{2}$. The above discussion can be formulated in the following lemma.

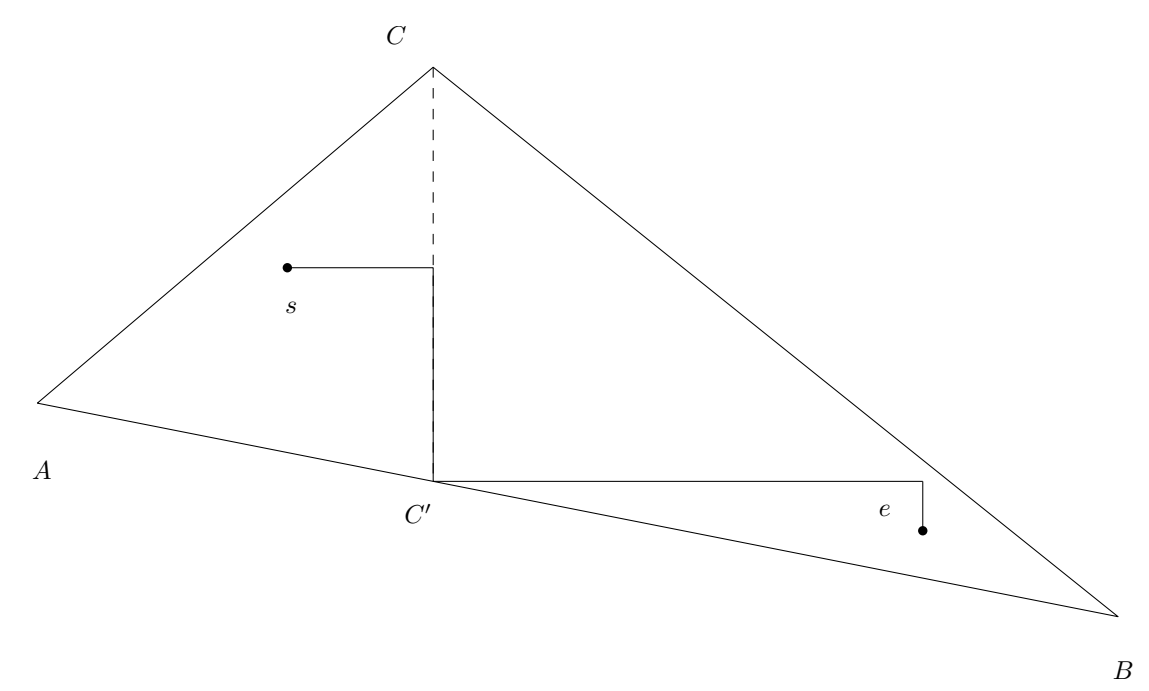

Figure 2.2.4: A line parallel to $y$ splits triangle to two sections each in position required for Lemma 11 and 12

Lemma 13. If $A B C$ is a triangle in $\mathbb{R}^{2}$ such that $x_{A} \leq x_{C} \leq x_{B}$ and $G_{\text {Dir }}$ is complete graph of size 4 over $\{x,-x, y,-y\}$, then for any given points $(s, e)$, algorithm 3 calculates a $G_{\text {Dir }}$ - admissible path of length $l$ connecting $s$ to e, where 


$$
l \leq 2\left(\mid\left\lfloor\log _{y_{C} / y_{C^{\prime}}}\left(1-\frac{y_{C}-y_{s}}{y_{C}}\right)\right\rfloor-\left\lfloor\log _{y_{C} / y_{C^{\prime}}}\left(1-\frac{y_{C}-y_{e}}{y_{C}}\right)\right\rfloor \pm 2\right) .
$$

\subsection{Finding a $G_{D i r^{-}}$-admissible path in a polygon}

Let $P$ be a polygonal environment then $P$ can be triangulated in $O(n \log (n))$ time with $O(n)$ storage (see [15] chapter 3 for details). Let $\left\{T_{1}, \cdots, T_{m}\right\}$ be such a triangulation for $P$. We define the weak dual graph $\mathbb{G}_{P}$ for the triangulation of $P$ in which the vertex set is $V_{\mathbb{G}_{P}}=\left\{T_{1}, \cdots, T_{m}\right\}$ and $\left(T_{i}, T_{j}\right) \in E_{\mathbb{G}_{P}}$ is an edge iff $T_{i}$ and $T_{j}$ share an edge. It can easily be observed that $\mathbb{G}_{P}$ is a planar graph. As a result the size of $V_{\mathbb{G}_{P}}$ and $E_{\mathbb{G}_{P}}$ are in order of $n$, the size of $P$ (since $m=O(n)$ ). It can easily be observed that each edge $e \in E_{\mathbb{G}_{P}}$ uniquely associated to an edge of the triangulation. For each edge $e \in E_{\mathbb{G}_{P}}$ we define $m_{e}$ to be the midpoint of the triangle edge that $e$ represents.

Based on triangulation $\left\{T_{1}, \cdots, T_{m}\right\}$ the following algorithm will find a $G_{D i r}$ admissible path between any two points $(s, e)$.

\section{Algorithm 4.}

Input: Polygonal environment $P$, its triangulation $\left\{T_{1}, \cdots, T_{m}\right\}$ and start and end points $(s, e)$

Output: $G_{D i r^{-}}$admissible path between s and e.

1. Find $T_{s}$ and $T_{e}$, the triangles which contain $s$ and e respectively.

2. If $T_{s}=T_{e}$ then run algorithm 3 for $T_{s}$ and $(s, e)$.

3. If $T_{s} \neq T_{e}$ then find a path $\Pi$ between $T_{i}$ and $T_{j}$ in $\mathbb{G}_{P}$. Assuming $|\Pi|=l$ we denote each vertex in this path by $\Pi(i)$ where $\Pi(0)=T_{s}$ and $\Pi(l)=T_{e}$. If such a path does not exist then report that there is no $G_{D i r^{-}}$admissible path between $s$ and $e$. 
4. Run algorithm 3 for triangle $\Pi(0)$ and $\left(s, m_{(\Pi(0), \Pi(1))}\right)$ and concatenate the result to $R$.

5. For each $\Pi(i)$ while $\Pi(i) \neq T_{e}$ run algorithm 3 for triangle $\Pi(i)$ and $\left(R_{E n d}, m_{(\Pi(i), \Pi(i+1))}\right)$ and concatenate the resulting path to $R . \quad\left(R_{E n d}\right.$ is the last point in $R$ ).

6. If $\Pi(i)=T_{e}$ run algorithm 3 for triangle $\Pi(l)$ and $\left(R_{E n d}, e\right)$ and concatenate the resulting path to $R$ and report $R$.

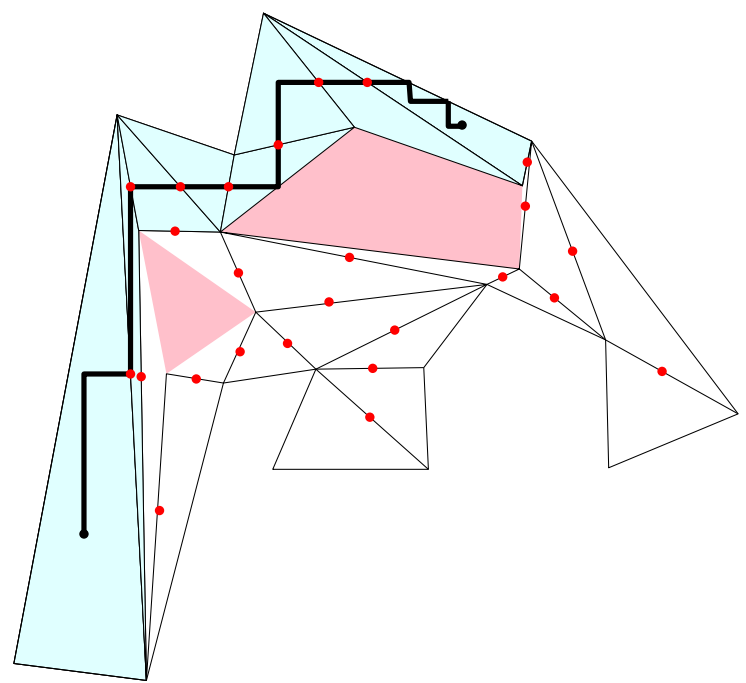

Figure 2.3.1: A sample run of Algorithm 4. The light blue section represents $\Pi$

Based on Lemma 13 it is clear that algorithm 4 finishes. It is also obvious that algorithm 4 generates a $G_{D i r^{-}}$admissible path. If there exists a path from $T_{s}$ to $T_{e}$ then Algorithm 4 will generate a $G_{D i r^{-}}$admissible path. Now if there exists a $G_{D i r^{-}}$ admissible path between $s$ and $e$ then this path corresponds to a walk $W$ on $\mathbb{G}_{P}$. This walk $W$ can be reduced to a path by removing all its cycles. Let $\Pi_{W}$ be such a path for $W$ then algorithm 4 generates a $G_{D i r}$-admissible path between $s$ and $e$.

This shows that Algorithm 4 generates a $G_{D i r^{-}}$admissible path between any given start and end points $(s, e)$ if and only if such a path exists. So we have 
Theorem 14. If $P$ is a polygonal environment in $\mathbb{R}^{2}$ and $G_{\text {Dir }}$ is a complete graph of size 4 over $\{x,-x, y,-y\}$, then for any given points $(e, e)$, Algorithm 4 calculates $a G_{\text {Dir }}$ - admissible path connecting s to e. 


\section{Chapter 3}

\section{Approximating a non-admissible path by a $G_{\text {Dir-admissible path }}$}

The problem of finding a piecewise linear path between two points in a polygonal environment has been extensively studied in two and three dimensions. It has been shown in [16] and [15] that this problem can be preprocessed in polynomial time and path queries can be answered by searching for the shortest path in the preprocessed structure. In this chapter we will assume that we are given a polygonal environment $P$ in $\mathbb{R}^{2}$, a direction transition graph $G_{D i r}(V, E)$, a pair of points $(s, e)$ and an obstacle avoiding path $R=\left(r_{0}=s, \cdots, r_{l}=e\right)$ in the interior of $P$. We will consider the problem of approximating $R$ by a $G_{D i r}$-admissible path between $s$ and $e$.

We first consider the case in which $G_{D i r}$ is a complete graph and show that $R$ can be approximated by a $G_{D i r}$-admissible path in this setting. Then we relax this restriction and study the problem for any strongly connected $G_{D i r}$ and we conclude that Problem 8 can be solved in this configuration. 


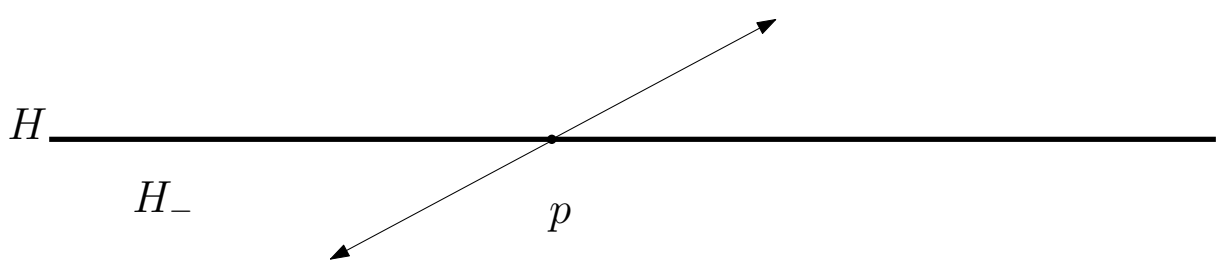

Figure 3.1.1: If $G_{D i r}$ is spanning then there is at least one $v \in V_{G_{D i r}}$ such that $v \notin H$. For such a $v$ either $v$ or $-v$ is in $H_{-}$

\subsection{Tubular neighborhoods and sleeves}

Lemma 15. If $G_{\text {Dir }}$ is symmetric and spanning then for a given half plane $H=$ $\{(x, y): a x+b y+c \leq 0\}$ and any point $p$ on the boundary of $H$ there exist $a$ vertex $v$ in $V_{G_{D i r}}$ such that $p+v=\left(x_{p}+x_{v}, y_{p}+y_{v}\right)$ lies in the open half plane $H$, $H_{-}=\{(x, y): a x+b y+c<0\}$. i.e $a\left(x_{p}+x_{v}\right)+b\left(y_{p}+y_{v}\right)+c<0$

Proof: Since $G_{D i r}$ is spanning there exists a vector $v \in V_{G_{D i r}}$ such that $p+v$ is not on the boundary of $H$. As illustrated in Figure 3.1.1, If $p+v$ is outside $H$ (i.e. $\left.a\left(x_{v}\right)+b\left(y_{v}\right)>0\right)$ then for $-v$ we have $a\left(x_{-v}\right)+b\left(y_{-v}\right)<0$. This proves that either $v$ of $-v$ has the desired property.

Definition 16. Let $a$ and $b$ be points in $P$ such that the line segment $\overline{a b}$ is in $P$. A tubular neighborhood for a point $z \in \overline{a b}$ along $\overline{a b}$ in $P$ is defined as,

$$
\operatorname{Box}(\overline{a b}, \lambda, z)=\{x+\beta \vec{c} ; x \in \overline{a b},\|z-x\|<\lambda,-\lambda<\beta<\lambda\}
$$

where $\vec{c}$ is the normal unit-vector of $\overline{a b}$. We denote its closure by

$$
\overline{\operatorname{Box}(\overline{a b}, \lambda, z)}=\{x+a \vec{c} ; x \in \overline{a b},\|z-x\| \leq \lambda,-\lambda \leq a \leq \lambda\} .
$$

We similarly define a tubular neighborhood along $\overline{a b}$ as

$$
\operatorname{Box}(\overline{a b}, \lambda)=\{x+a \vec{c} ; x \in \overline{a b},-\lambda<a<\lambda\} .
$$


Figure 3.1.2 depicts the tubular neighborhood of a point along a line and a line segment. 
i)

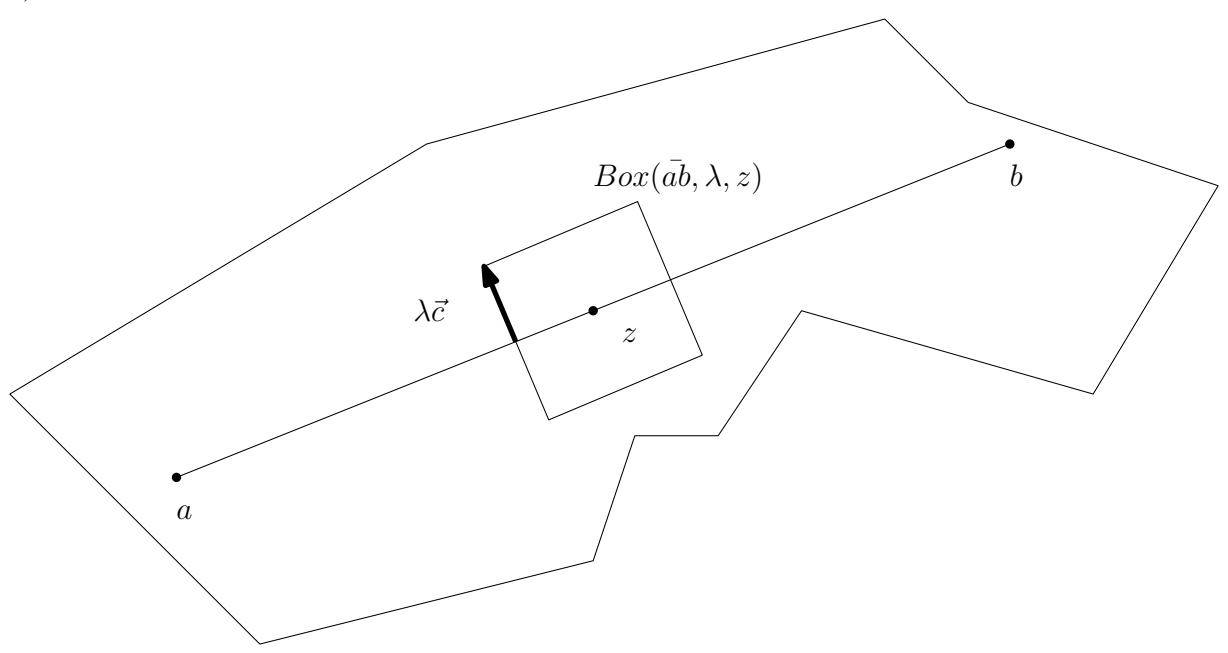

ii)

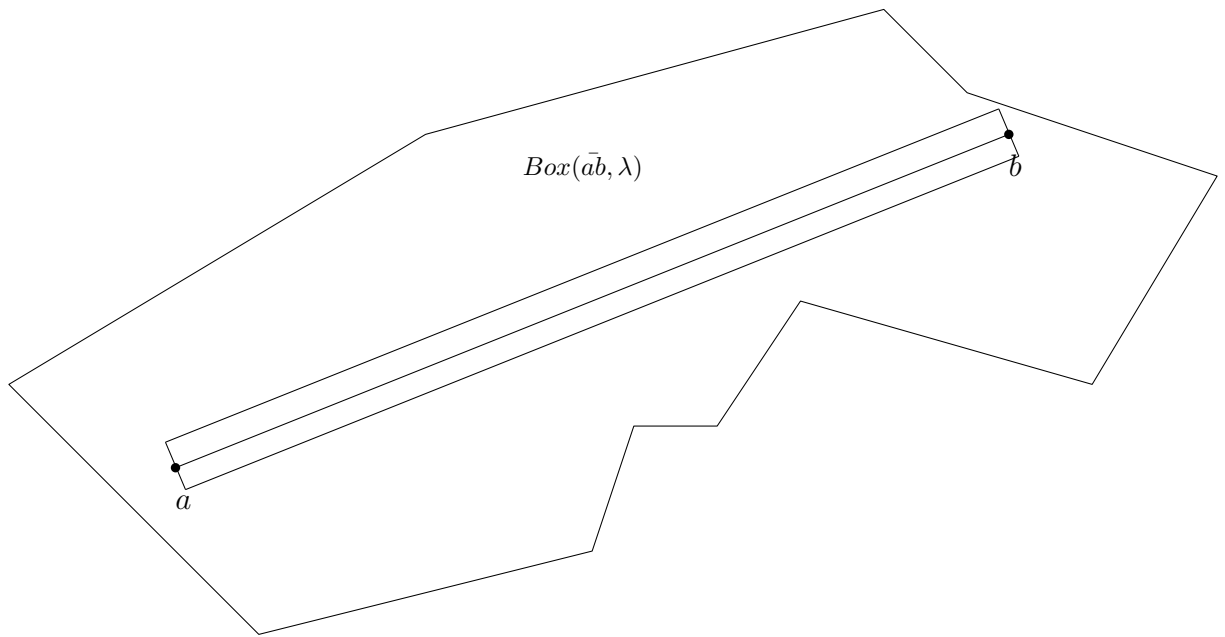

Figure 3.1.2: i) Tubular neighborhood of $z$ along $\overline{a b}$ ii) tubular neighborhood of $\overline{a b}$ 
Let $R=\left(r_{0}=s, \cdots, r_{l}=e\right)$ be a piecewise linear path in $P$ which connect $s$ and $e$. For each $i$, let $\lambda_{i}$ be the minimum orthogonal distance of $\overline{r_{i} r_{i+1}}$ from the boundary $\partial P$ of $P$, i.e,

$$
\lambda_{i}=\min \left\{D_{c}(x): x \in \overline{r_{i} r_{i+1}}\right\}
$$

where

$$
D_{c}(x)=\min \{\alpha: x+\alpha c \in \partial P\} \text {, }
$$

and $c$ is a unit orthogonal vector to $\overline{r_{i} r_{i+1}}$. Compactness of $\overline{r_{i} r_{i+1}}$ guarantees that such a minimum exists. Any tubular neighborhood $\operatorname{Box}\left(\overline{r_{i} r_{i+1}}, \lambda_{i} / 2\right)$ of $\overline{r_{i} r_{i+1}}$ resides entirely in the interior of $P$.

This will define a collection of tubular neighborhoods for line segments of $R$,

$$
\left\{\overline{\operatorname{Box}\left(\overline{r_{i} r_{i+1}}, \lambda_{i} / 2\right)} ; 0 \leq i<l\right\}
$$

which are completely in $P$. We call this collection a sleeve for $R$. The following theorem can be concluded from the above observation.

Theorem 17. For any piecewise linear path $R$ inside the interior of a polygon $P$, there exist a sleeve for $R$ such that its closure is completely in the interior of $P$.

Next we will show that for a given polygonal environment $P$ (possibly with some holes), a pair of points $(s, e)$ and a piecewise linear path $R$ between $s$ and $e$, Algorithm 5 computes a sleeve for $R$ which consists of tubular neighborhoods of the line segments of the path.

The basic idea is to find the orthogonal distance between each line segment to the boundary of the polygonal environment $P$. Basically Algorithm 5 loops through the line segments $\overline{r_{i} r_{i+1}}$ of piecewise linear path $R$. For each line segment:

1. It finds an orthonormal base $\left(c_{1}, c_{2}\right)$ at $r_{i} . c_{1}$ is in the direction of $\overline{r_{i} r_{i+1}}$ and $c_{2}$ is orthogonal to $c_{1}$. 


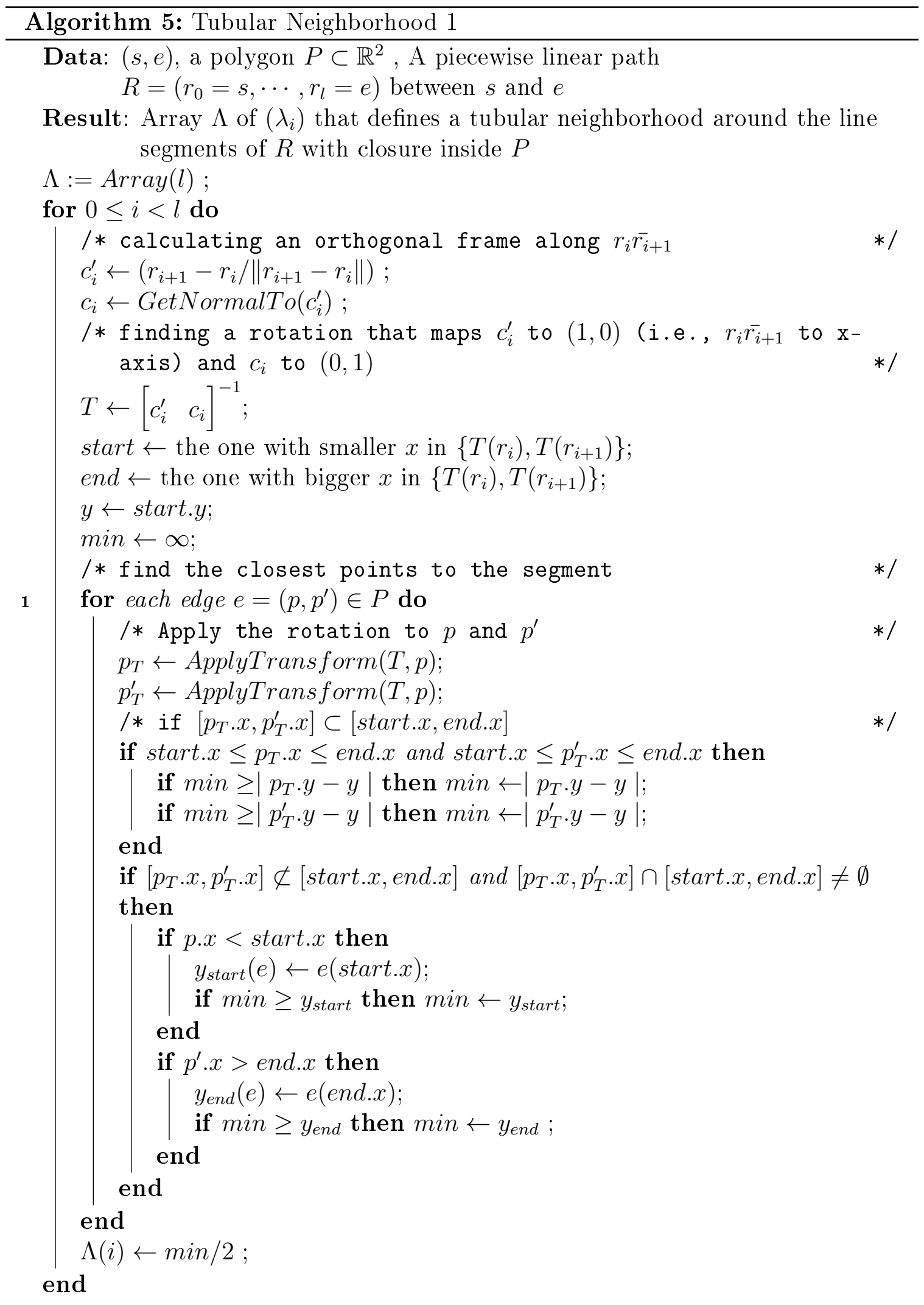




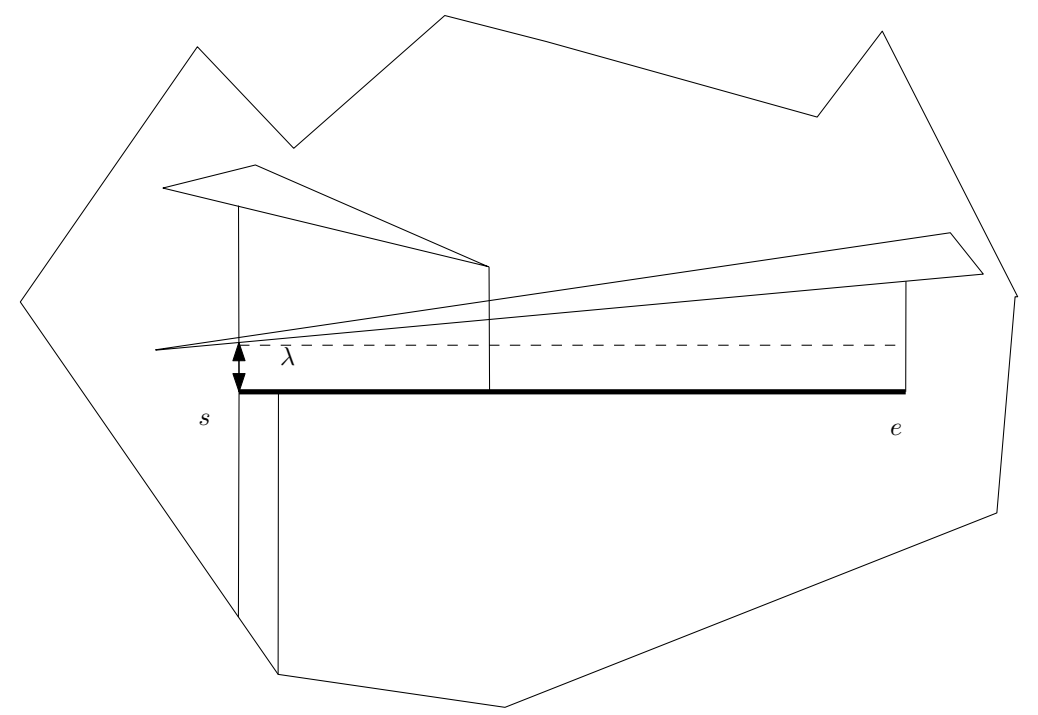

Figure 3.1.3: Sample run of Algorithm 5 for the path $R=(s, e)$.

2. The whole environment is rotated and translated in a such way that $\left(c_{1}, c_{2}\right)$ maps to $((1,0),(0,1))$. As a result $r_{i}$ is mapped to $(0,0)$ and $r_{i+1}$ to $\left(0, \alpha_{i+1}\right)$. (Figure 3.1.3)

3. For each edge $\overline{p p^{\prime}} \in P_{T}$ we consider the projection of $\overline{p p^{\prime}}$ on the $x$-axis, $\pi_{x}\left(\overline{p p^{\prime}}\right)=$ $\left[p_{x}, p_{x}^{\prime}\right]$. Let $I=\left[p_{x}, p_{x}^{\prime}\right] \cap\left[0, \alpha_{i+1}\right]$. We calculated the height of the end points of $I$ when $\overline{p p^{\prime}}$ is considered as a linear function over $I$ and keep track of minimum of such heights. Since $I$ is convex and $\overline{p p^{\prime}} \in P_{T}$ considered as a line over $I$ is both convex and concave the height of the end points have the extreme values. So the minimum of these extreme values defines the orthogonal distance of $\overline{r_{i} r_{i+1}}$ with $P$.

4. We report half of the minimum calculated in the above step as $\Lambda_{i}$.

Theorem 18. Algorithm 5 computes a sleeve for $R$ in $O(\ln )$ time.

Proof: Algorithm 5 is iterating over the line segments of $R$ and for each line segment $\overline{r_{i} r_{i+1}}$ it finds a $\Lambda(i)$ such that the tubular neighborhood $\overline{\operatorname{Box}\left(\overline{r_{i}, r_{i+1}}, \lambda\right)}$ resides in $P$. To do so for each line segment $\overline{r_{i} r_{i+1}}$, the algorithm finds the biggest $\lambda_{i}$ such that 
$\operatorname{Box}\left(\overline{r_{i}, r_{i+1}}, \lambda_{i}\right)$ is in $P . \lambda_{i}$ is equal to

$$
\min \left\{\operatorname{Dist}(x, x+\alpha \vec{c}) ; x+\alpha \vec{c} \in \operatorname{boundry}(P) \& x \in \overline{r_{i} r_{i+1}}\right\}
$$

where $\vec{c}$ is a unit vector perpendicular to $\overline{r_{i} r_{i+1}}$. It can be observed that such a minimum only happens on the extreme points of $\overline{r_{i} r_{i+1}}$ or points of $P$.

As the first step to facilitate the calculation, we rotate $P$ in such a way that $\overline{r_{i}, r_{i+1}}$ becomes parallel to the $x$-axis. The rotation needs to be applied to the $n$ vertices of $P$, which takes $O(n)$ time. On the next step we go through all edges $e$ of $P$ and categorize them by how their projection on the $x$-axis intersects the projection of $\overline{r_{i} r_{i+1}}$ on the $x$-axis. This can be done just by examining the $x$-coordinate of each vertex of $e$ against the $x$-coordinate of transformed $r_{i}$ and $r_{i+1}$. Since there are $n$ edges, this operation takes $O(n)$ time. If the projection of $e$ on the $x$-axis is included in the projection of $\overline{r_{i} r_{i+1}}$ then the minimum will happen on the extreme points of $e$ hence its end vertices. If the projection of $e$ on the $x$-axis has a non-empty intersection with the projection of $\overline{r_{i} r_{i+1}}$ then the minimum can happen at the end points of $\overline{r_{i} r_{i+1}}$ or the end points of $e$. To examine the intersection of the projection of $e$ we examine the $x$-coordinates of the rotated edge against the end points of $\overline{r_{i} r_{i+1}}$ after the rotation is applied. The categorization of the edges can be done in constant time.

Based on the discussion above the whole process will take $O(\ln )$ to complete.

\subsection{Approximating a line segment if $G_{D i r}$ is the com- plete graph}

In this section we study the problem of approximating a piecewise linear path $R=$ $\left(r_{0}=s, \cdots, r_{l}=e\right)$ in polygon $P$, by a $G_{D i r}$-admissible path when $G_{D i r}$ is the 

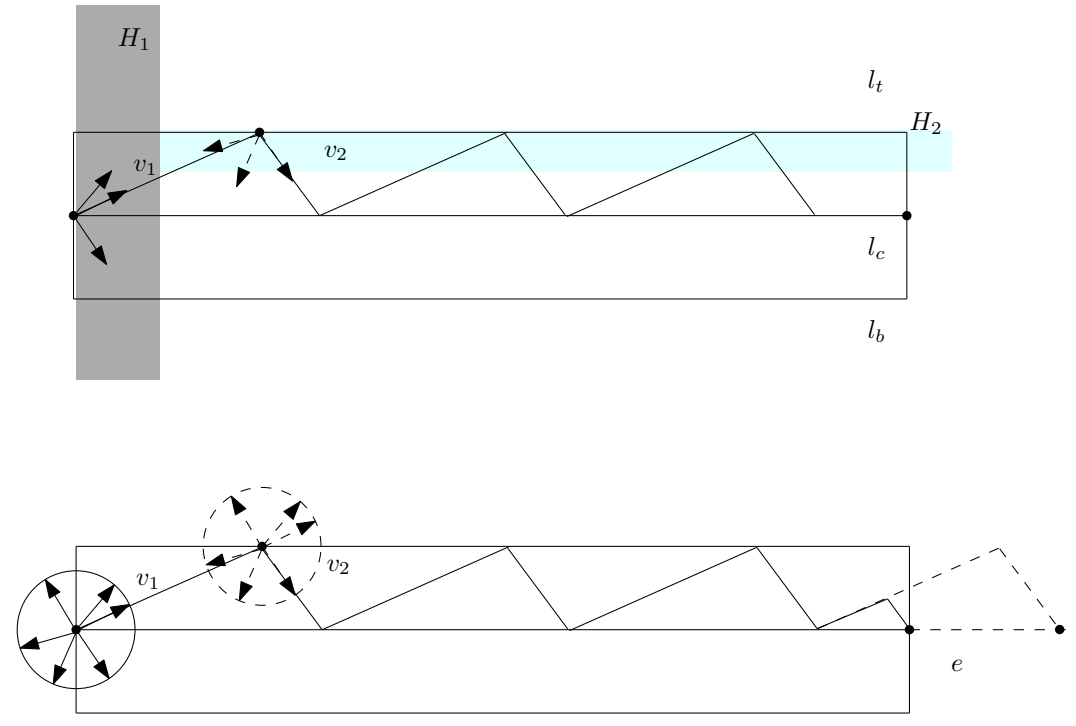

Figure 3.2.1: Sample run of Algorithm ZigZag

complete graph. We calculate a tubular neighborhood $\operatorname{Box}\left(\overline{r_{i}, r_{i+1}}, \lambda_{i}\right)$ for each $\overline{r_{i} r_{i+1}}$ and approximate $\overline{r_{i} r_{i+1}}$ in $\operatorname{Box}\left(\bar{r}_{i}, r_{i+1}, \lambda_{i}\right)$ by a $G_{D i r}$-admissible path, as illustrated in Figure 3.2.1.

Now let us assume that $G_{D i r}$ is a complete graph and is spanning and symmetric. Assume the members of the set $V$ of admissible directions are sorted by their slopes. We will denote the set of all rays out of a point $x$ in directions in $V$, by $V_{x}$. Given a line segment $\overline{s e}$ and a tubular neighborhood $\operatorname{Box}(\overline{s e}, \lambda)$, let $l_{t}$ and $l_{b}$ be the top and bottom lines supporting $\operatorname{Box}(\overline{s e}, \lambda)$ and $l_{C}$ the centerline that contains $\overline{s e}$.

Definition 19. Let $v_{1}$ and $v_{2}$ be two vectors in $\mathbb{R}^{2}$. We say that $v_{1} \leq_{\alpha} v_{2}$ if and only if either $v_{2}$ is to the left of $O$ and $v_{1}$ is to the right of $O$, or $v_{1}$ and $v_{2}$ are both on the same side of $O$ and $v_{2}$ is to the left of $v_{1}$. Here, $O$ is an arbitrary reference vector.

It can easily be observed that $v_{1} \leq_{\alpha} v_{2}$ if and only if $v_{2}$ has a greater signed angle with $O_{v}$ than $v_{1}$. 


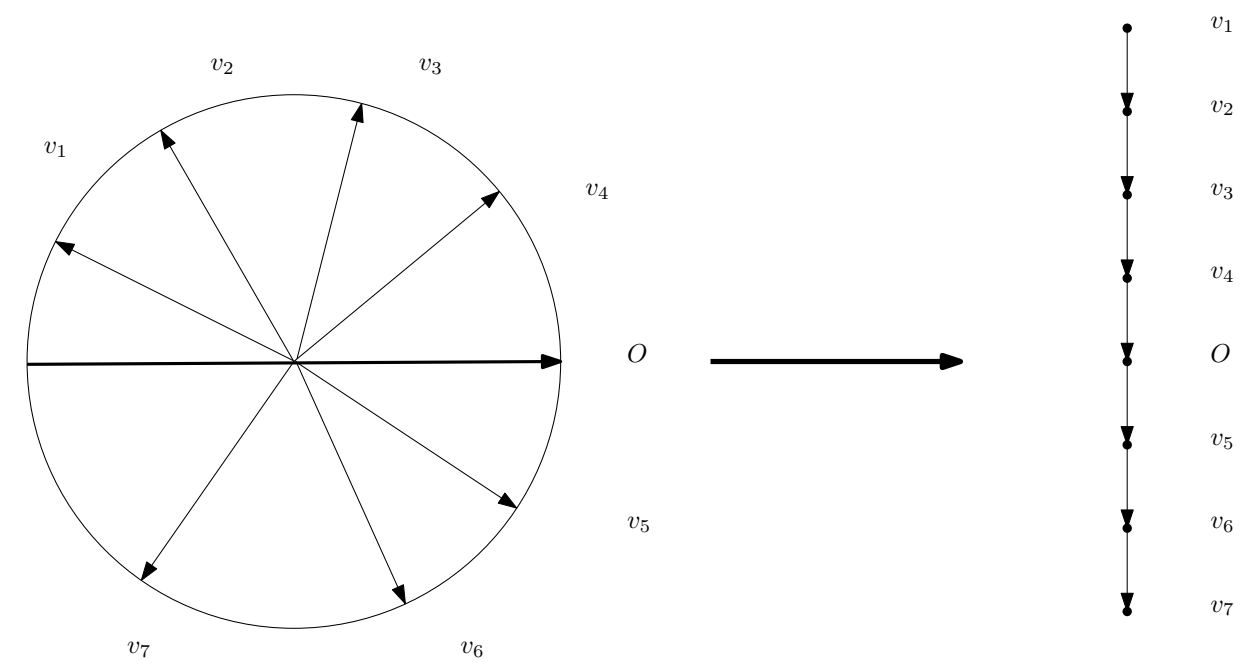

Figure 3.2.2: i) Illustration of or angular ordering imposed by left turn test

Let us assume that the direction of $\overrightarrow{s e}$ is not in $G_{D i r}$ and consider the half space $H^{+}=\{x: c \cdot(s-x) \geq 0)$, where $c$ is a normal vector of $\overrightarrow{s e}$. This half space contains $\overline{s e}$ and its boundary is perpendicular to $\overline{s e}$. By Lemma 15, $V_{s} \cap H^{+}$is not empty and has cardinality at least two, where $V_{s}$ is the translation of vectors in $V$ to point $s$. Let $v_{1}$ be the vector in $V$ with minimum angular difference with $\overline{s e}$. We assume that $v_{1}$ is to the left of $\overrightarrow{s e} \subset l_{c}$, so any ray starting from $l_{c}$ meets $l_{t}$. The result below is dually repeatable for the case that $v_{1}$ is to the right of $\overrightarrow{s e}$.

As shown in Figure 3.2.1, we move from $s$ along $v_{1}$ until we reach $l_{t}$ at $x_{1}$. Let $L_{s \vec{x}_{1}}^{+}$be the half plane on the right of $s \vec{x}_{1}$ which contains $\overline{s e} . V_{x_{1}} \cap L_{s \vec{x}_{1}}^{+}$has at least one element by Lemma 15 . Let $L_{t}^{+}$be the half plane to the right of $l_{t}$ which contains $\operatorname{Box}(\overline{s e}, \lambda)$. We show that $V_{x_{1}} \cap L_{s \vec{x}_{1}}^{+} \cap L_{t}^{+}$also has at least one element. This easily follows from the fact that $L_{t}^{+} \cap L_{s \vec{x}_{1}}^{+}$cannot contain any ray from $V_{x_{1}}$ because $v_{1}$ has minimum angular difference with $l_{c}$ and hence $l_{t}$.

We choose $v_{2} \in V_{x_{1}} \cap L_{s \vec{x}_{1}}^{+} \cap L_{t}^{+}$to have minimum angular difference with $l_{t}$. Now we continue from $x_{1}$ along with $v_{2}$ until we reach $l_{c}$ again at $x_{2}$. The minimality in the selection of $v_{1}$ and $v_{2}$ guarantees that $x_{2}-s=\beta(e-s)$ for some $\beta>0$.

This process can be continued further by looking at the half space $L_{x_{1} x_{2}}^{-}$to the 
left of $x_{1} \vec{x}_{2}$ and $L_{c}^{-}$, the half space to the left of $\overrightarrow{s e}$ which contains $v_{1}$, and selecting the direction $v_{3}$ with minimal angular difference with $l_{c}$. The next lemma shows that $v_{3}=v_{1}$.

Lemma 20. Assuming $v_{1}, v_{2}$ and $v_{3}$ have been selected as above. Then $v_{1}=v_{3}$.

Proof: $v_{3}$ belongs to $L_{x_{1} \vec{x}_{2}}^{-} \cap L_{c}^{-} \cap V_{x_{2}}$ and is the right most member of it. Based on the assumption above about $v_{1}$, we have $v_{1} \in H^{+} \cap L_{c}^{-} \cap V_{s} \subset L_{x_{1} \vec{x}_{2}}^{-} \cap L_{c}^{-} \cap V_{x_{2}}$ but since every element of $L_{x_{1} \vec{x}_{2}}^{-} \cap L_{c}^{-} \cap V_{x_{2}}$ is to the left or equal to an element of $H^{+} \cap L_{c}^{-} \cap V_{s}$ (i.e., greater or equal in term of angle)the minimum element will be equal, hence $v_{1}=v_{3}$.

The following lemma will also help to simplify the process of calculating $v_{1}$ and $v_{2}$.

Lemma 21. For a given pair of start and end points $(s, e)$ if $\overrightarrow{s e}$ does not match the direction of any of the vectors in $V_{G_{D i r}}, v_{1}$ and $v_{2}$ can be calculated by finding the placement of $\overrightarrow{s e}$ in the ordered list $\left(V_{G_{D i r}}, \leq_{\alpha}\right)$. The placement of $\overrightarrow{s e}$ in the list gives us two elements $v_{\max }=\max \left\{v \in V_{\text {dir }} ; v \leq_{\alpha} \overrightarrow{s e}\right\}$ and $v_{\text {min }}=\min \left\{v \in V_{G_{D i r}} ; \overrightarrow{s e} \leq_{\alpha} v\right\}$. If the absolute value of the signed angle between $v_{\max }$ and $\overrightarrow{\mathrm{se}}$ is greater than the angle between $v_{\min }$ and $\overrightarrow{s e}$, then $v_{1}=v_{\min }$ and $v_{2}=v_{\max }$, and vise versa otherwise.

Proof: The proof of this lemma is rather obvious based on how $v_{1}$ and $v_{2}$ are selected in the above process. Assume the absolute value of the angle between $v_{\max }$ and $\overrightarrow{s e}$ is greater than angle between $v_{\text {min }}$ and $\overrightarrow{s e}$. Let $v_{1}$ be the closest element in $V_{G_{D i r}} \cap H^{+} \cap L_{c}^{+}$to $e-s$ angularly. $v_{\min }$ is in $L_{c}^{+}$by definition, so $v_{\min } \leq_{\alpha} v_{1}$. We also have $v_{\text {min }} \in H^{+}$since otherwise $v_{1} \leq_{\alpha} c \leq_{\alpha} v_{\text {min }}$ which contradicts the above result so $v_{\min }=v_{1}$. Similarly it can be concluded that $v_{2}=v_{\max }$. For the case that the absolute value of the angle between $v_{\max }$ and $\overrightarrow{s e}$ is less than the angle between $v_{\min }$ and $\overrightarrow{s e}$, all the results will be dual of the above, i.e., $v_{\max }=v_{1}$ and $v_{\min }=v_{2}$. 
Algorithm Zigzag is approximating a line segment with an $G_{D i r^{-}}$admissible path. We can list the steps of it as follows:

1. Set $H:=\{c \mid c .(e-s) \geq 0\}$.

2. Define $v_{1}$ to be the closest vector in $V_{s} \cap H$ to the direction of $\overline{s e}$ according to the ordering $\leq_{\alpha}$

3. Based on whether $v_{1} \leq_{\alpha} \overline{s e}$ or $\overline{s e} \leq_{\alpha} v_{1}$, continue from $s$ in the direction of $v_{1}$ until we meet $l_{b}$ or $l_{t}$ at $z \vec{i} g$.

4. Calculate an inward normal vector $n$, for example for $l_{c}$, and set $H=\{c \mid$ $c \cdot(n) \geq 0\} \cdot n$ is calculated in such a way that $H$ always contains $l_{c}$.

5. Define $v_{2}$ to be the closest vector in $V_{s} \cap H$ to the direction of $\overline{s e}$ according to the ordering $\leq_{\alpha}$.

6. Continue from $z i g$ in the direction of $v_{2}$ until we meet $l_{c}$ in $z a g$.

7. Continue alternating in the directions of $v_{1}$ and $v_{2}$ and zigzag between $l_{t}$ (or $l_{b}$ depending on $v_{1}$ ) and $l_{c}$ until zag meets $l_{c}$ past $e$.

8. Scale and adjust the last zig zag so it meets $l_{c}$ at $e$. 


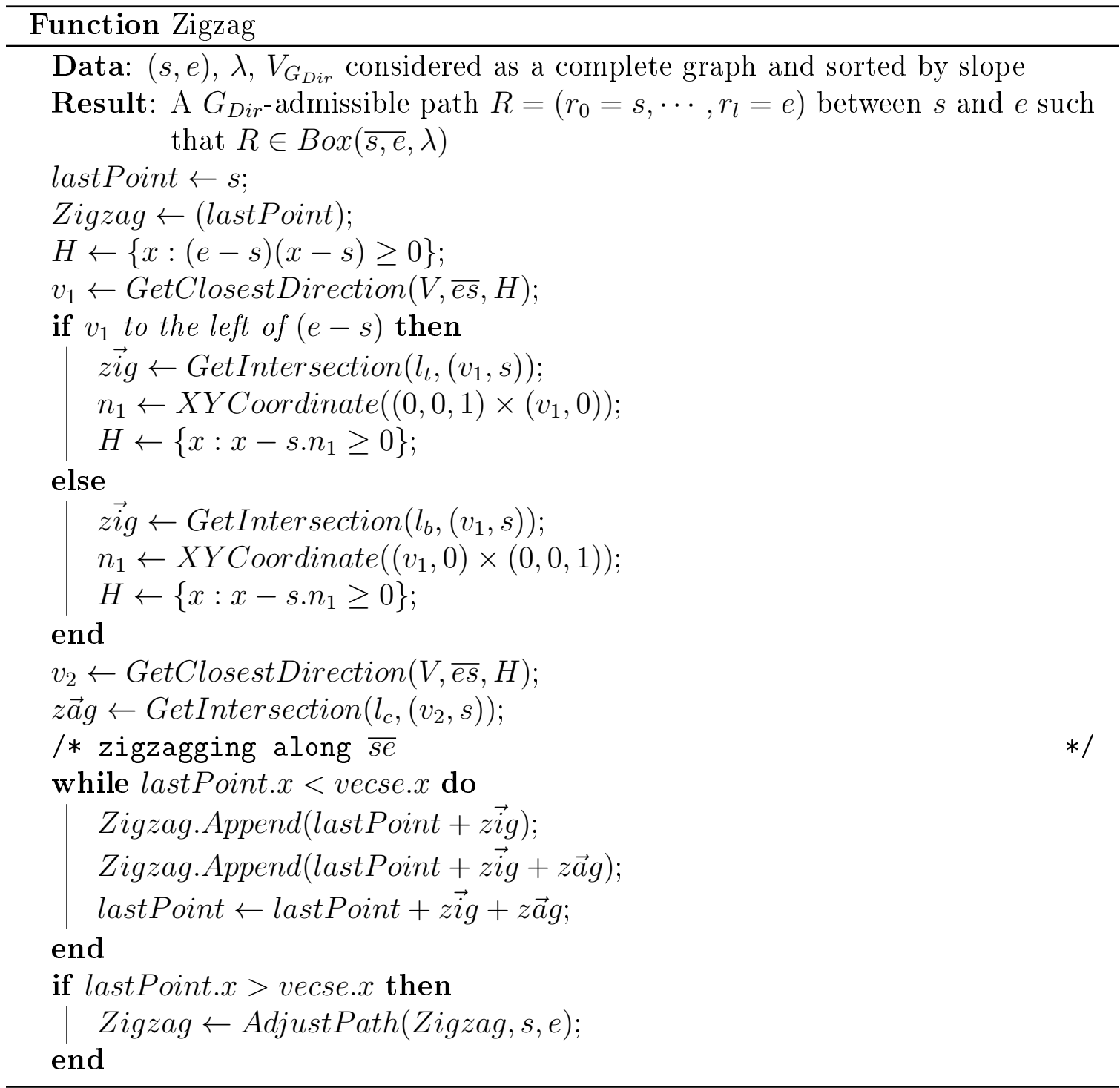

The following lemma proves the correctness of Algorithm Zigzag.

Lemma 22. If $G_{\text {Dir }}$ is complete, spanning, and symmetric, $(s, e)$ is a pair of points and Box $(\overline{s e}, \lambda)$ is a tubular neighborhood of $\overline{s e}$ in $\mathbb{R}^{2}$ then there exists a $G_{\text {Dir- }}$ Admissible path $R$ between $s$ and e which is completely inside Box(se, $\lambda)$. Algorithm Zigzag computes such a path in $O\left(\log (m)+\|s-e\| / \lambda \cot \left(\angle v_{i+1}-v_{i}\right)\right)$ time. The length of $R$ satisfies $\|R\| \leq\|s-e\|\left(1+1 / \cot \left(\angle v_{i+1}-v_{i}\right)\right)$. Here, $v_{i}$ and $v_{i+1}$ are two consecutive vectors (with respect to $\leq_{\alpha}$ ) with maximum angular difference. 


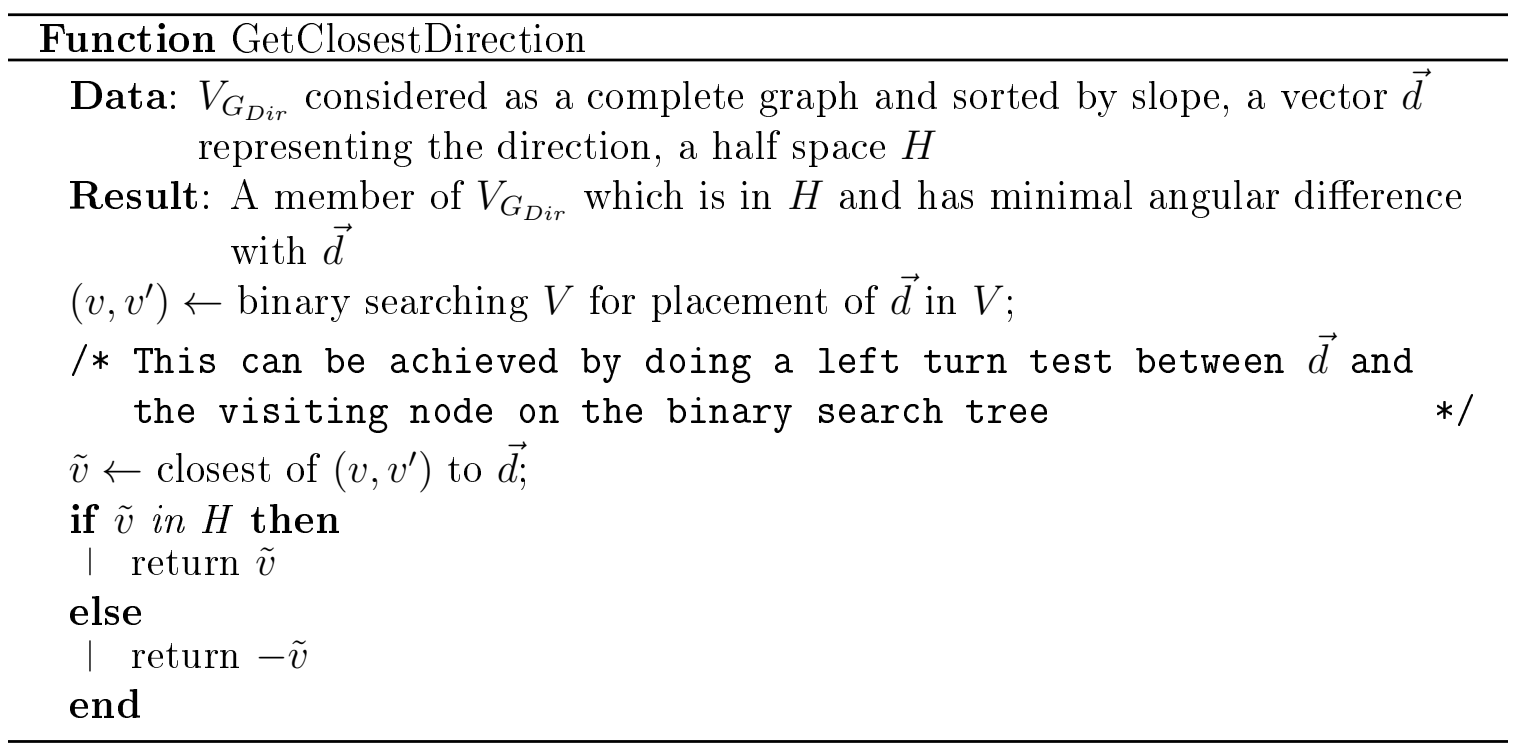

\section{Function GetIntersection}

Data: $\left(\overrightarrow{d_{1}}, \overrightarrow{b_{1}}\right)$ representing the first line, $\left(\overrightarrow{d_{2}}, \overrightarrow{b_{2}}\right)$ representing the second line Result: the intersection point of line 1 and line 2 $x_{\overrightarrow{d_{1}}} \leftarrow$ x-coordinate of $\overrightarrow{d_{1}}$ $y_{\overrightarrow{d_{1}}} \leftarrow$ y-coordinate of $\overrightarrow{d_{1}}$ $x_{\overrightarrow{d_{2}}} \leftarrow$ x-coordinate of $\overrightarrow{d_{2}}$; $y_{\overrightarrow{d_{2}}} \leftarrow$ y-coordinate of $\overrightarrow{d_{2}}$ $x_{\overrightarrow{b_{1}}} \leftarrow \mathrm{x}$-coordinate of $\overrightarrow{b_{1}}$; $y_{\overrightarrow{b_{1}}} \leftarrow$ y-coordinate of $\overrightarrow{b_{1}}$; $x_{\overrightarrow{b_{2}}} \leftarrow$ x-coordinate of $\overrightarrow{b_{2}}$; $y_{\overrightarrow{b_{2}}} \leftarrow$ y-coordinate of $\overrightarrow{b_{2}}$;

$\alpha \leftarrow \frac{\left(y_{b_{2}}-y_{b_{1}}\right) x_{d_{1}}-\left(x_{b_{2}}-x_{b_{1}}\right) y_{d_{1}}}{x_{d_{2}} y_{d_{1}}-x_{d_{1}} y_{d_{2}}} ;$ Return $\overrightarrow{b_{2}}+\alpha \overrightarrow{d_{2}}$;

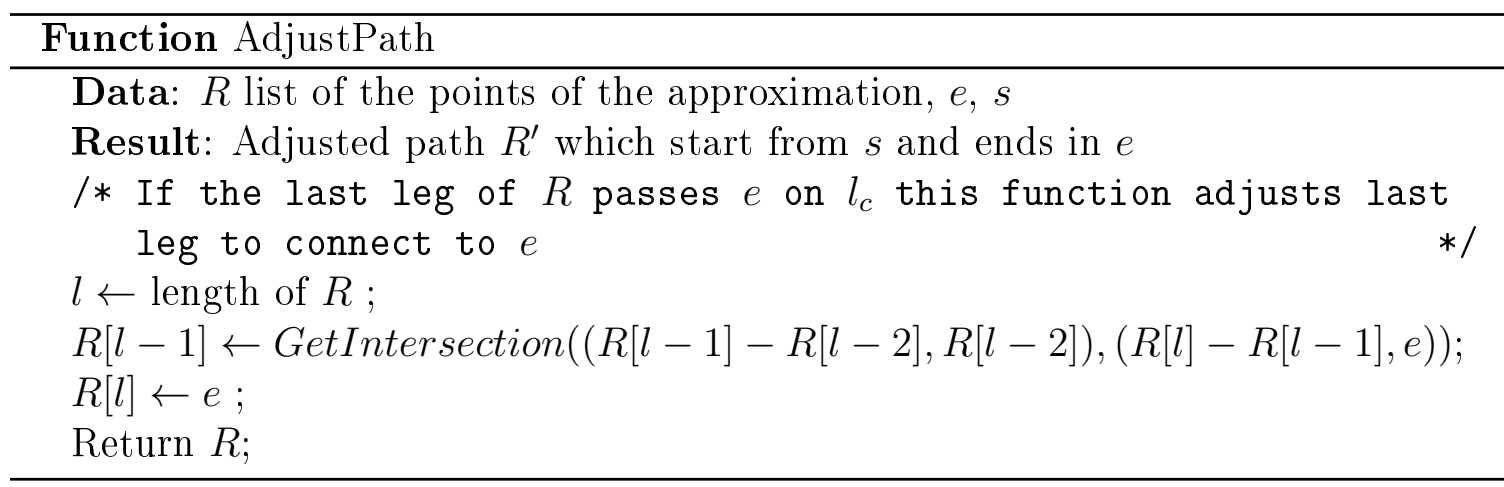


Proof: It is obvious that the path generated by algorithm Zigzag is $G_{D i r}$-admissible. To start we show that the algorithm finishes. The only iteration in algorithm Zigzag happens in its sole while loop in which two points zig and zag are added to the approximating path Zigzag in each iteration. Since zag is always on $l_{c}$ by definition, in order to check if we have reached the end point $e$ we only need to compare the $x$-coordinate of lastPoint against the $x$-coordinate of $e$. This is because for each value of $z a g$ there is a constant $\alpha$ such that $z a g=s+\alpha(e-s)$. The value of lastPoint increases in each iteration to lastPoint $+z i g+z a g=$ lastPoint $+\beta(e-s) /\|e-s\|$ for some $\beta>0$. We have

$$
\beta=\lambda\left(\frac{\cos \left(\angle v_{1}-\angle \overrightarrow{s e}\right)}{\sin \left(\angle v_{1}-\angle \overrightarrow{s e}\right)}-\frac{\cos \left(\angle v_{2}-\angle \overrightarrow{s e}\right)}{\sin \left(\angle v_{2}-\angle \overrightarrow{s e}\right)}\right)
$$

i.e.,

$$
\beta=\lambda\left(\frac{\sin \left(\left(\angle v_{1}-\angle \overrightarrow{s e}\right)-\left(\angle v_{2}-\angle \overrightarrow{s e}\right)\right)}{\sin \left(\angle v_{1}-\angle \overrightarrow{s e}\right) \sin \left(\angle v_{2}-\angle \overrightarrow{s e}\right)}\right)
$$

As a result we have:

$$
\beta=\lambda\left(\frac{\sin \left(\angle v_{1}-\angle v_{2}\right)}{\sin \left(\angle v_{1}-\angle \overrightarrow{s e}\right) \sin \left(\angle v_{2}-\angle \overrightarrow{s e}\right)}\right)
$$

Now we find out for a given $v_{1}$ and $v_{2}$, which angle of $\overrightarrow{s e}$ minimize the value of $\beta$.To do so we examine the zeros of the derivative of $\beta$ in terms of the angle of $\overrightarrow{s e}$, $d \beta / d \angle \overrightarrow{s e}$ is

$$
-\frac{\sin \left(\angle v_{1}-\angle v_{2}\right)\left(\sin \left(\angle v_{1}-\angle \overrightarrow{s e}\right) \cos \left(\angle v_{2}-\angle \overrightarrow{s e}\right)+\sin \left(\angle v_{1}-\angle \overrightarrow{s e}\right) \cos \left(\angle v_{2}-\angle \overrightarrow{s e}\right)\right)}{\left(\sin \left(\angle v_{1}-\angle \overrightarrow{s e}\right) \sin \left(\angle v_{2}-\angle \overrightarrow{s e}\right)\right)^{2}},
$$


which can be simplified to

$$
-\frac{\sin \left(\angle v_{1}-\angle v_{2}\right) \sin \left(\angle v_{1}+\angle v_{1}-2 \angle \overrightarrow{s e}\right)}{\left(\sin \left(\angle v_{1}-\angle \overrightarrow{s e}\right) \sin \left(\angle v_{2}-\angle \overrightarrow{s e}\right)\right)^{2}}
$$

Based on the above identity $\angle \overrightarrow{s e}=\left(\angle v_{1}+\angle v_{1}\right) / 2$ is a zero of $d \beta / d \angle \overrightarrow{s e}$. Since the denominator of $d \beta / d \angle \overrightarrow{s e}$ is always positive and based on the sign of nominator, it can be concluded that $\left(\angle v_{1}+\angle v_{1}\right) / 2$ is a local minima.

By putting back the minimum value into (3.2.1) we have

$$
\beta=2 \lambda\left(\frac{\left.\cos \left(\angle v_{1}-\angle v_{2}\right) / 2\right)}{\sin \left(\left(\angle v_{1}-\angle v_{2}\right) / 2\right)}\right)=2 \lambda \cot \left(\left(\angle v_{2}-\angle v_{1}\right) / 2\right)
$$

Since cot is decreasing, $\beta$ acquires its minimum value when the difference between $v_{1}$ and $v_{2}$ is maximum.

Based on Lemma 21, $v_{1}$ and $v_{2}$ are always two consecutive vectors in $V_{G_{D i r}}$ ordered by $\leq_{\alpha}$. Let $i$ be an index for which the value $v_{i+1}-v_{i}$ is maximum. Then we have $\beta \geq 2 \lambda \cot \left(\angle v_{i+1}-v_{i} / 2\right)$. So the while loop will add at least $2 \lambda \cot \left(\angle v_{i+1}-v_{i}\right)$ to lastPoint $-s$. Therefore the while loop finishes at most after $\|s-e\| / 2 \lambda \cot \left(\angle v_{i+1}-v_{i}\right)$ iterations. The number of edges in Zigzag is at least twice the number of iterations of the while loop, i.e., $\|s-e\| / \lambda \cot \left(\angle v_{i+1}-v_{i}\right)$.

It takes $O(\log m)$ to find $v_{1}$ and $v_{2}$ in the sorted list of directions $V_{G_{D i r}}$. Plus the time spent in the while loop this proves our claim about the complexity of algorithm Zigzag.

It also can be observed that for each edge $e_{i}$ of $Z i g z a g$ we have $\left\|e_{i}\right\| \leq\left\|f_{i}\right\|+\lambda$ where $f_{i}$ is the orthogonal projection of $e_{i}$ on $\overline{s e}$. Therefore $\sum\left\|e_{i}\right\| \leq \sum\left\|f_{i}\right\|+l . \lambda$ where $l$ is the number of edges in Zigzag. By substituting the value of $l$ from the above we have $\sum\left\|e_{i}\right\| \leq\|s-e\|\left(1+\tan \left(\angle v_{i+1}-v_{i}\right)\right)$ 


\subsection{Approximating a line segment if $G_{D i r}$ is strongly connected}

Now we draw our attention to the more general case where $G_{\text {Dir }}$ is only strongly connected. If $G_{D i r}$ is strongly connected then for any two directions $v_{i}$ and $v_{j}$ in $V_{G_{D i r}}$ there exists a path from $v_{i}$ to $v_{j}$ and one from $v_{j}$ to $v_{i}$.

We define an $\varepsilon$-padding of $B o x(\overline{s e}, \lambda)$ to be the box around $B o x(\overline{s e}, \lambda)$ with edges at distance $\varepsilon$ of the edges of $\operatorname{Box}(\overline{s e}, \lambda)$, as illustrated in Figure 3.3.1. More precisely,

$$
\operatorname{Box}_{\varepsilon}(\overline{s e}, \lambda)=\operatorname{Box}\left(s_{\varepsilon}^{-} e_{\varepsilon}, \lambda+\varepsilon\right)
$$

where $s_{\varepsilon}=s-\varepsilon(e-s /\|e-s\|)$ and $e_{\varepsilon}=e+\varepsilon(e-s /\|e-s\|)$. We won't need to calculate $s_{\varepsilon}$ or $e_{\varepsilon}$ in our algorithm picking a small enough $\varepsilon$ will suffice.

Lemma 23. Let $x \in B(s, \varepsilon)$, be a point inside the ball with center $s$ and radius $\varepsilon$, $v$ a vector, and $l$ a line not parallel to $v$. Let $l_{v}$ and $l_{v}^{\prime}$ be two lines tangent to $B(s, \varepsilon)$ and parallel to $v$. Then the line $l_{x}$ passing through $x$ parallel to $v$, meets $l$ between $l_{v}$ and $l_{v}^{\prime}$.

Proof: This results from the fact that $l_{v}$ and $l_{v}^{\prime}$ and $l_{x}$ are all parallel to each other.

First we show that if $G_{\text {Dir }}$ is strongly connected, spanning and symmetric, then the weak version of admissible path planning is possible.

The new algorithm ZigzagEx has a template very similar to algorithm Zigzag. First we find $v_{1}$ and $v_{2}$ as in algorithm Zigzag. The only difference is that here we cannot switch from $v_{1}$ to $v_{2}$ and vise versa, instead we should go through the paths between $v_{1}$ and $v_{2}$. Based on Theorem 10, we can control how far away we wander from the intersection of each leg of zigzags and $l_{t}\left(\right.$ or $\left.l_{b}\right)$ or $l_{c}$. Given a path $\left(v_{1}, \cdots, v_{n}\right) \in G_{\text {Dir }}$ and a point $c$ and a number $\beta$, function BuildPath is building a 

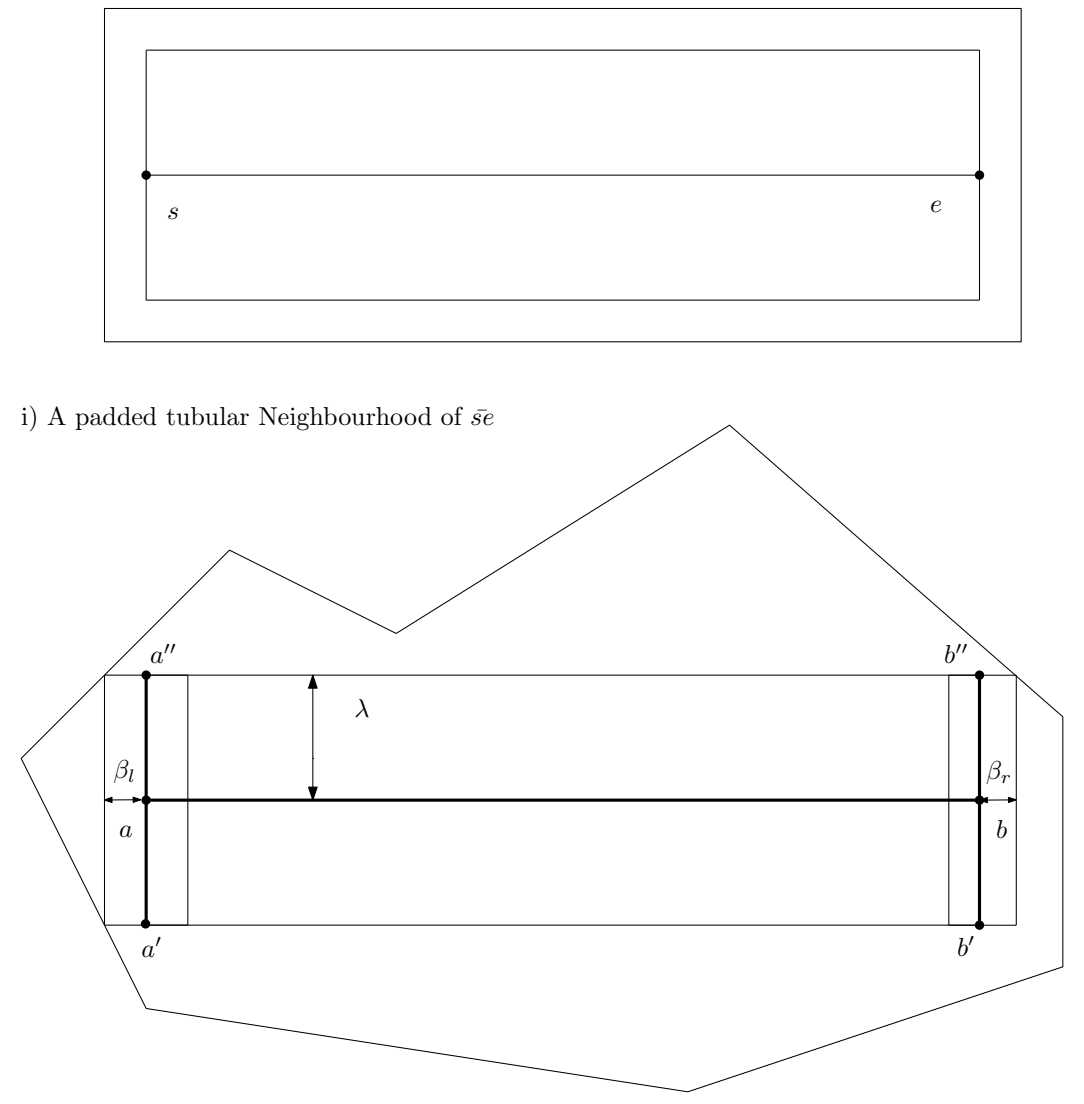

ii) Findling a tubularr neighbourhood

Figure 3.3.1: $\varepsilon$ padding of $\operatorname{Box}(\overline{s e}, \lambda)$ 


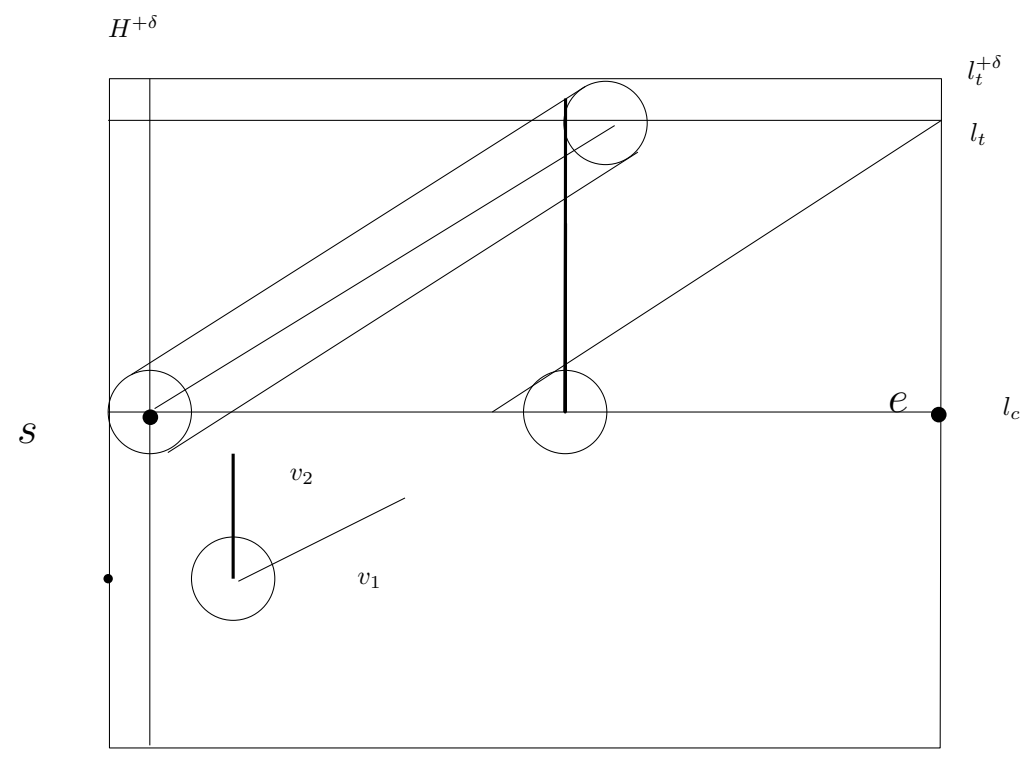

Figure 3.3.2: Approximating a line segment when $G_{D i r}$ is strongly connected.

piecewise linear path $\left(c, c+\beta v_{1}=c_{1}, \cdots, c_{n}+\beta v_{n}\right)$ which by Lemma 10 ends in a ball of radius $\beta W$ around $c$, where $W$ is the length of $\left(v_{1}, \cdots, v_{n}\right)$ with Euclidean metric. If $\left(v_{1}, \cdots, v_{n}\right)$ is the shortest path between $v_{1}$ and $v_{n}$ then the path generated by BuildPath is in a ball with radius $\beta \operatorname{Diam}\left(G_{\text {Dir }}\right)$, where $\operatorname{Diam}\left(G_{\text {Dir }}\right)$ is the diameter of Graph $G_{D i r}$, i.e., the maximum length of all shortest paths in $G_{\text {Dir }}$. For a given $\varepsilon$, if $\beta=\varepsilon / \operatorname{Diam}\left(G_{\text {Dir }}\right)$, the path generated by BuildPath will always be in a ball of radius $\varepsilon$ around $c$.

To prove that the method of algorithm Zigzag still works, we show that if $\varepsilon$ is selected small enough, the section of the path generated by application of BuildPath does not wander off the padded tubular neighborhood and also Algorithm ZigzagEx still moves forward by a constant distance on $l_{c}$ in each iteration. The first is achieved easily by selecting $\varepsilon<\delta$.

Figures 3.3.2 and 3.3.3 depict an iteration of Algorithm ZigzagEx. In each iteration we start from the last point of Zigzag, which we denote by $s_{i}$ and is initialized by the starting point $s$. we apply BuildPath with $P_{v_{2} v_{1}}$ to generate a path $\left(s_{i}, \cdots, c_{i}^{v_{1}}, c_{i}^{v_{1}}+\right.$ $\left.\beta v_{1}\right)$ in the $\varepsilon$-neighborhood of the starting point $s_{i}$. Then we start from $c_{i}^{v_{1}}$ in direction 


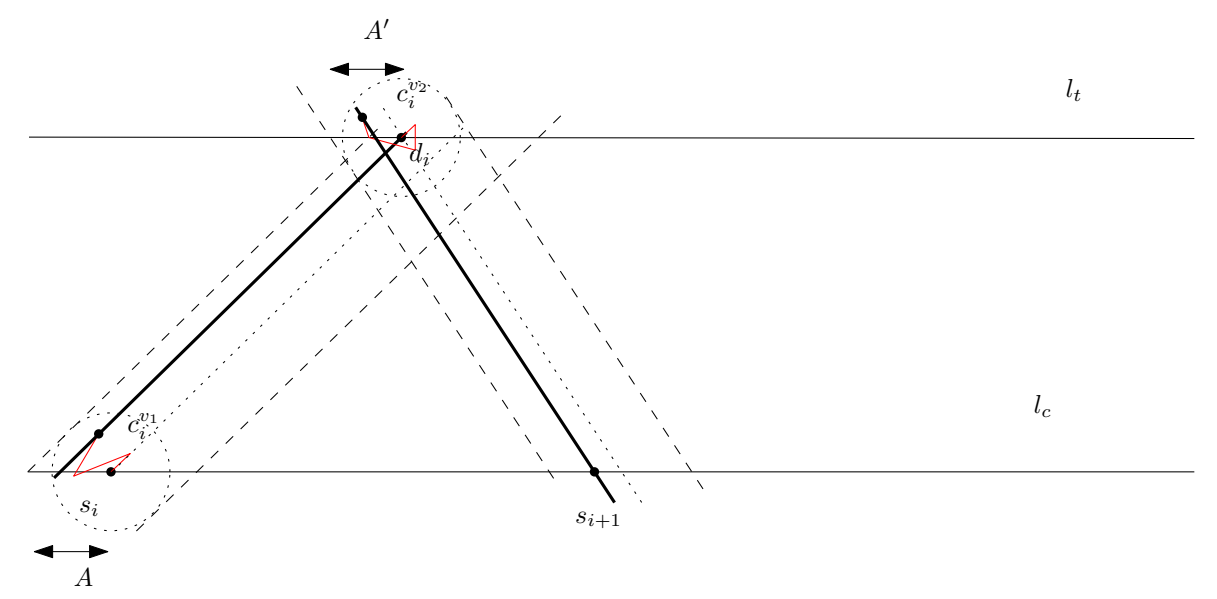

Figure 3.3.3: An iteration of the Crisscross function

of $v_{1}$ and continue until it intersect with $l_{t}$ (or $l_{b}$ depending on $v_{1}$ ) at $d_{i}$. Reapplying BuildPath with $P_{v_{2} v_{1}}$, generates a path $\left(d_{i}, \cdots, c_{i}^{v_{2}}, c_{i}^{v_{2}}+\beta v_{2}\right)$ in the $\varepsilon$-neighborhood of $d_{i}$. Then continue from $c_{i}^{v_{2}}$ in the direction of $v_{2}$ until it reaches $l_{c}$ at $s_{i+1}$.

Now let us compare the above iteration with an iteration of Algorithm Zigzag. We start from $s_{i}$. Follow the direction of $v_{1}$ until we meet $l_{t}$ at $d_{i}^{\prime}$ and continue from there in the direction $v_{2}$ until we intersect $l_{c}$ in $s_{i+1}^{\prime}$. By Lemma 22, $\left\|s_{i}-s_{i+1}^{\prime}\right\|$ is always greater or equal to $\lambda \cot \left(\operatorname{Max}_{d i f} / 2\right)$, where $\operatorname{Max}_{d i f}$ is the maximum angular difference between two consecutive directions in $V_{G_{D i r}}$ ordered by $\leq_{\alpha}$. Let $z_{1}$ and $z_{2}$ be the two lines representing the envelope of the boundary of the $\varepsilon$-disks around $s_{i}+\beta v_{1}$ for any $\beta$. $z_{1}$ and $z_{2}$ are tangent to all $\varepsilon$-disks around $s_{i}+\beta v_{1}$ and hence parallel to the vector $d_{i}^{\prime}-s_{i}$. Since the vector $d_{i}-c_{i}^{v_{1}}$ is also parallel to $d_{i}^{\prime}-s_{i}$ then by Lemma $23 d_{i}$ is located between $m_{1}=z_{1} \cap l_{t}$ and $z_{2} \cap l_{t}$. As a result we have $\left\|d_{i}-d_{i}^{\prime}\right\|<A_{v_{1}}=\left\|m_{1}-d_{i}^{\prime}\right\|$ (see Figure 3.3.5). If $f_{d_{i}}$ is the intersection of $d_{i}+\beta v_{2}$ with $l_{c}$, parallelity gives us $\left\|s_{i+1}^{\prime}-f_{d_{i}}\right\|<A_{v_{1}}$. Based on the same argument we can also show that $\left\|s_{i+1}-f_{d_{i}}\right\|<A_{v_{2}}$, and again Lemma 23 gives us $\left\|s_{i+1}-s_{i+}^{\prime}\right\|<A_{v_{1}}+A_{v_{2}}$. From above we have $\left\|s_{i}-s_{i+1}\right\|>\left\|s_{i}-s_{i+1}^{\prime}\right\|-A_{v_{1}}-A_{v_{2}}$ and hence $\left\|s_{i}-s_{i+1}\right\|>2 \lambda \cot \left(\operatorname{Max}_{d i f} / 2\right)-A_{v_{1}}-A_{v_{2}}$.

Let us focus on $v_{1}$ as it is depicted in figure 3.3.4. Let $A=A_{v_{1}}$. From the triangular 


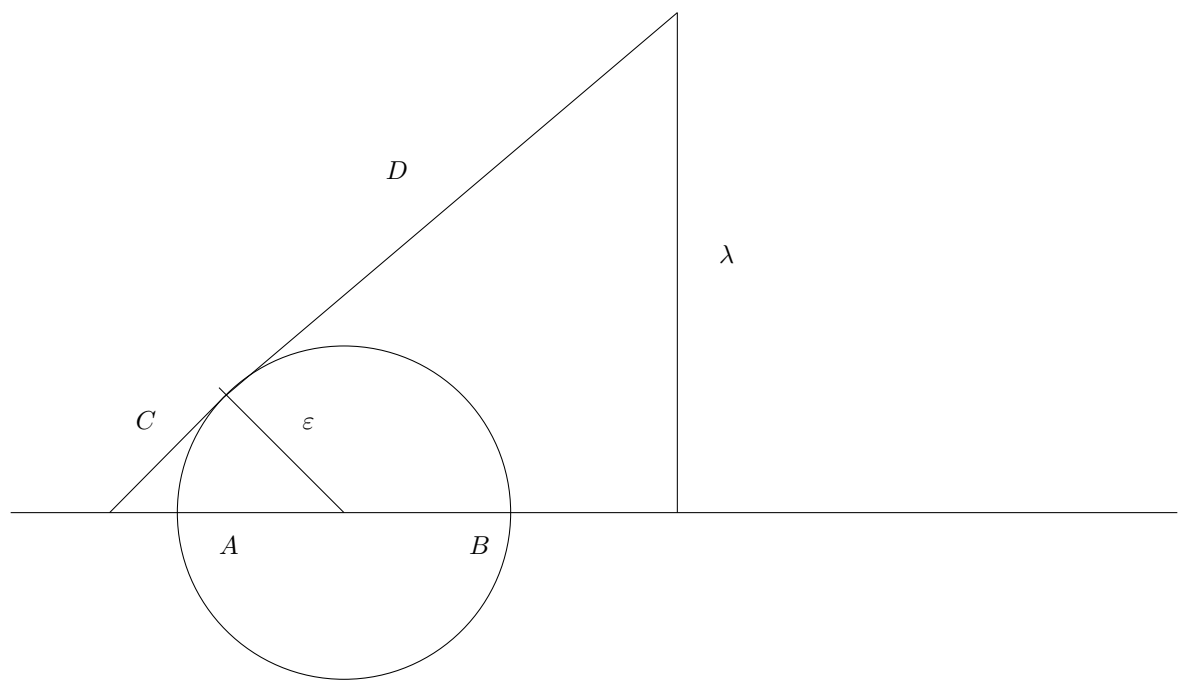

Figure 3.3.4: $\varepsilon /(\lambda)=A /(C+D)=C /(A+B)$

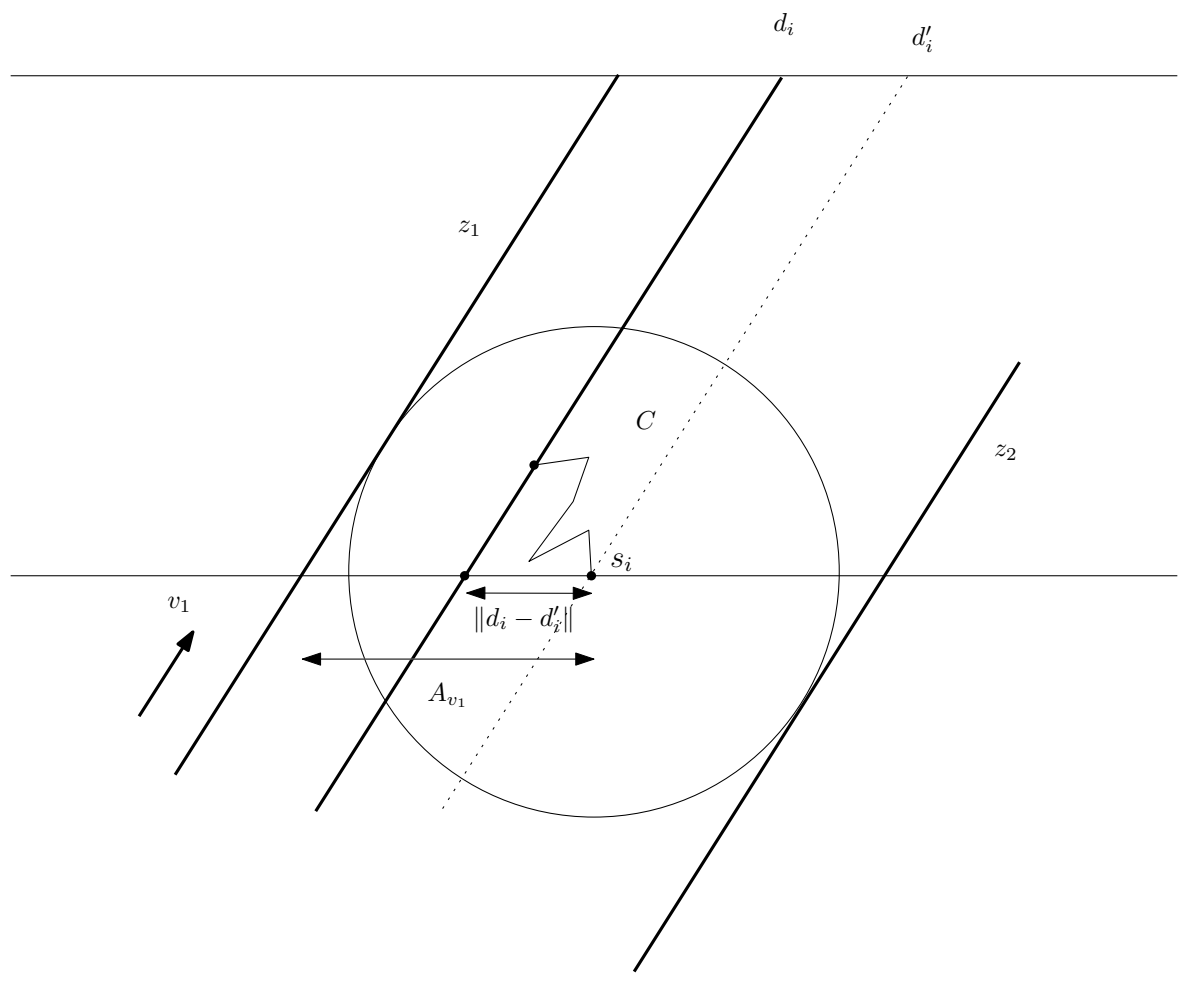

Figure 3.3.5: An $\varepsilon$ deviation from from starting point $s$ 
similarities in Figure 3.3.4 we have $\varepsilon / \lambda=C /(A+B)$ hence $C=\varepsilon(A+B) / \lambda$. If we calculate $A$ in term of $C$ and $\varepsilon$ we have $A^{2}=C^{2}+\varepsilon^{2}$ so $A^{2}=(\varepsilon(A+B) / \lambda)^{2}+\varepsilon^{2}$, from which we have $A=\sqrt{(\varepsilon(A+B) / \lambda)^{2}+\varepsilon^{2}}$ or $A=\varepsilon / \lambda \sqrt{\left.((A+B))^{2}+\lambda\right)^{2}}$. Since both $(A+B)$ and $\lambda$ are greater than zero we have $(A+B)^{2}+\lambda^{2} \leq(A+B)^{2}+\lambda^{2}+2(A+B) \lambda$ and hence $A \leq \varepsilon / \lambda((A+B)+\lambda)$. Unless the algorithm finishes in one iteration, we can assume that $A+B \leq\|s-e\|$ and hence $A \leq \varepsilon((\|s-e\| / \lambda)+1)$. This inequality is independent of the selection of $v_{1}$ and applies to $v_{2}$ as well. To avoid the complexity of calculating $\|s-e\|,(e-s) \cdot(e-s)=\|s-e\|^{2}$ can be used as well. Assume that $\varepsilon$ is chosen such that

$$
K:=\lambda \cot \left(\left(\angle v_{1}-\angle v_{2}\right) / 2\right)-\varepsilon(\|s-e\| / \lambda+1)>0
$$

Now let us assume algorithm ZigzagEx finishes in more than 1 iterations (otherwise it is a trivial case) and $\varepsilon$ is selected small enough such that (3.3.1) holds. Then in each iteration algorithm ZigzagEx moves forward along $\overline{e s}$ at least as far as $K$. This ensures the termination of the algorithm in at most $\|s-e\| / K$ iterations.

The next difference between algorithm Zigzag and ZigzagEx is the path adjustment step. In algorithm Zigzag, the adjustment step is only adjusting the last leg of the approximated path but in this case it is more complex because more than two edges are involved when $e$ is in the $\varepsilon$-neighborhood of Zigzag.LastPoint. To overcome that adjustment step, the algorithm scales down the computed path Zigzag by a factor of $k=e . x / Z i g z a g . L a s t P o i n t() . x$ as formulated in function AdjustPath.

Lemma 24. Let $G_{\text {Dir }}$ be strongly connected, spanning, and symmetric, $(s, e)$ a pair of points and $\operatorname{Box}_{\delta}(\overline{s e}, \lambda)$ a $\delta$-padding of the tubular neighborhood of $\overline{s e}$ in $\mathbb{R}^{2}$. If $\varepsilon$ is selected small enough then algorithm ZigzagEx computes a $G_{\text {Dir-admissible path }}$ between $s$ and e which resides inside $\operatorname{Box}_{\delta}(\overline{s e}, \lambda)$.

It can be observed that if $\varepsilon$ is selected in such a way that inequality (3.3.1) 


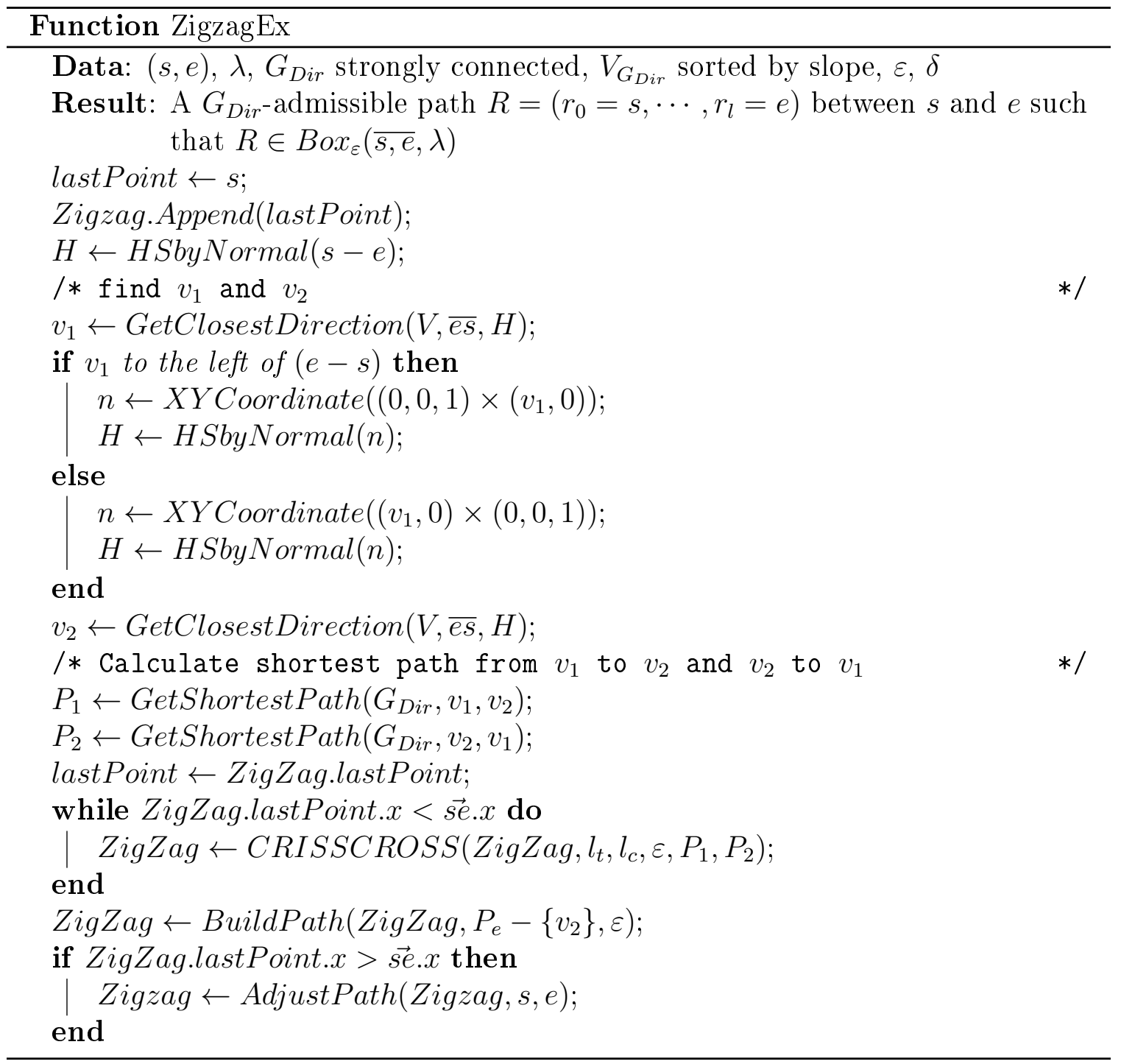

$l_{t}$

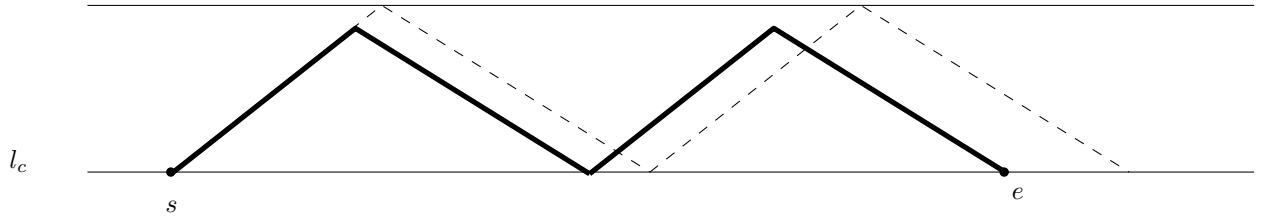

$l_{b}$

Figure 3.3.6: Dotted path generated by algorithm ZigzagEx before adjustment. Heavier path illustrated the same path after adjustment. 


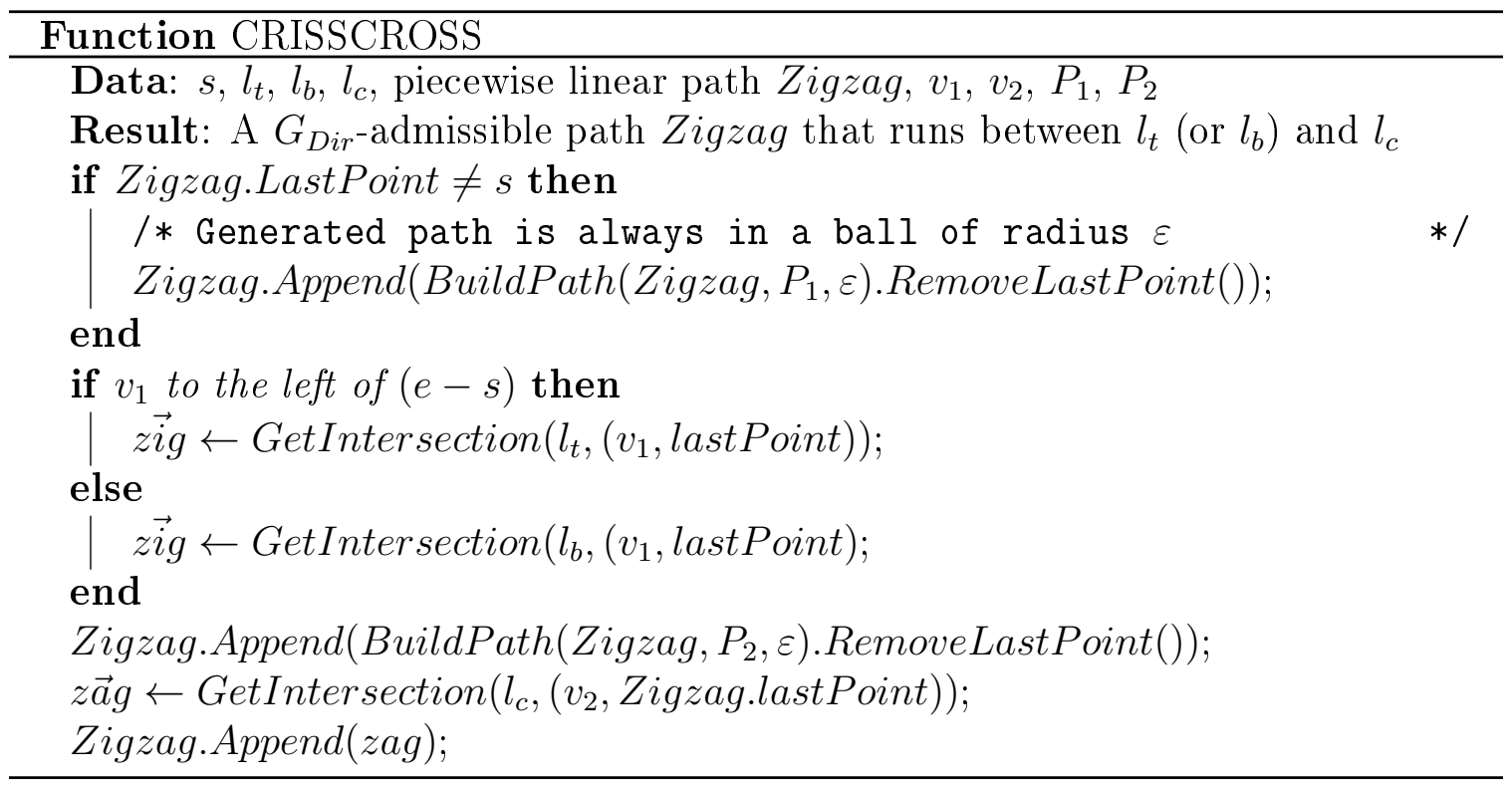

\section{Function BuildPath}

Data: Center $c$, path $\left(v_{1}, \cdots, v_{n}\right)$ in $G_{d i r}$, and a scaling factor $\beta$.

Result: A piecewise linear $G_{D i r}$-admissible path $\Pi$ based on $\left(v_{1}, \cdots, v_{n}\right)$ and starting from $c$.

Add $c$ to $\Pi$;

$p \leftarrow c$;

for $1 \leq i \leq n$ do

$p \leftarrow p+\beta \cdot v_{i}$

Add $p$ to $\Pi$;

end

\section{Function BuildPathRevers}

Data: Center $c$, path $\left(v_{1}, \cdots, v_{n}\right)$ in $G_{d i r}$, and a scaling factor $\beta$.

Result: A piecewise linear $G_{D i r}$-admissible path $\Pi$ based on $\left(v_{1}, \cdots, v_{n}\right)$ and starting from $c$.

$P \leftarrow$ Revers of $\left(-v_{1}, \cdots,-v_{n}\right)$;

Call BuildPath with $c, P$ and $\beta$; 


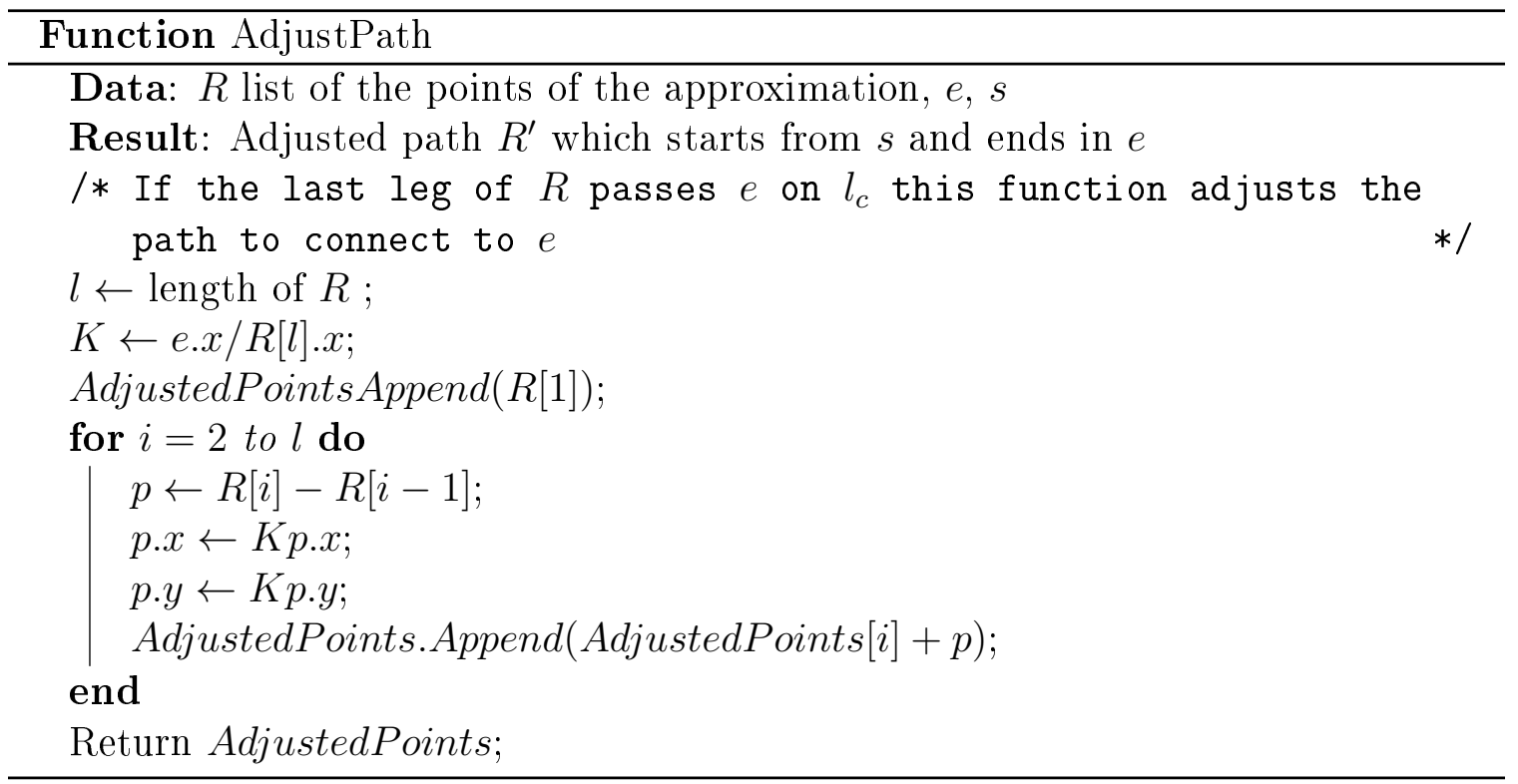

holds then the same $\varepsilon$ will also work for any $\left(e^{\prime}, s^{\prime}\right)$ with $\left\|e^{\prime}-s^{\prime}\right\| \leq\|e-s\|$. We can use this property to show that the strong form of the problem is also solvable. Let $\left(v_{s}, v_{e}\right)$ be two given start and end directions and let $P_{v_{s} v_{1}}$ and $P_{v_{2} v_{e}}$ be the shortest paths from $v_{s}$ to $v_{1}$ and $v_{2}$ to $v_{e}$ respectively. Starting from $e$ we run function BuildPathRevers to get the piecewise linear path $\left(c_{v_{2}}, \cdots, e\right)$ based on $P_{v_{2} v_{e}}$. This is done by traversing $P_{v_{2} v_{e}}$ in reverse order and building the sequence of points $p[i]=$ $p[i+1]-\beta / \operatorname{Diam}\left(G_{D i r}\right) P_{v_{2} v_{e}}[i+1]$ where $p[l]=e$ and $l$ is the length of $P_{v_{2} v_{e}}$. This will put $p[1]=c_{v_{2}}$ in the $\beta$-neighborhood of $e$.

Next we use BuildPath to build a path for $P_{v_{s} v_{1}}$ starting from $s$ in the $\beta$ neighborhood of $s$. Let $q$ be the second last point of this path which means we can move forward in the direction of $v_{1}$ from this point. Starting from $q$, we will follow $v_{1}$ until we reach $l_{t}$ or $l_{c}$. If we reach $l_{c}$ first we choose the intersection point as the new starting point $s^{\prime}$, otherwise from the intersection point with $l_{t}$, we follow the direction of $v_{2}$ back to $l_{c}$ until we meet $l_{c}$ at the new starting point $s^{\prime}$.

Similarly for $\left(c_{v_{2}}, \cdots, e\right)$, starting from $c_{v_{2}}$ we follow the direction of $-v_{2}$ until we reach either $l_{c}$ or $l_{t}$. If we reach $l_{c}$ first and the intersection point is between $s$ and 
$e$ we will choose the new end point $e^{\prime}$ to be this intersection point. Otherwise from the intersection point with $l_{t}$ we follow $-v_{1}$ until we reach $l_{c}$ and we will choose the intersection as the new end point $e^{\prime}$. Now if $\beta$ is less than $\varepsilon$ we can apply algorithm ZigzagEx to find a $G_{D i r^{-}}$admissible path between $s^{\prime}$ and $e^{\prime}$ and hence between $s$ and $e$, starting in direction $v_{s}$ and ending in direction $v_{e}$.

Lemma 25. Let $G_{\text {Dir }}$ be strongly connected, spanning, and symmetric, $(s, e)$ a pair of points, $\left(v_{s}, v_{e}\right)$ a pair of start and end directions and $B_{0} x_{\delta}(\overline{s e}, \lambda)$ a $\delta$-padding of the tubular neighborhood of $\overline{s e}$ in $\mathbb{R}^{2}$. If $\varepsilon$ is selected small enough then Algorithm

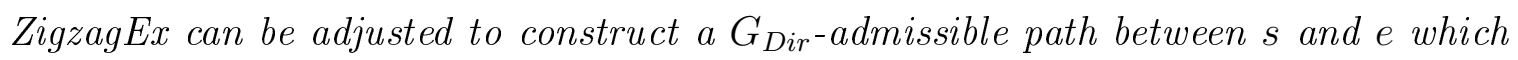
is inside $\operatorname{Box}_{\delta}(\overline{s e}, \lambda)$.

\subsection{Approximating a path with a $G_{D i r}$-admissible path}

We conclude this chapter by showing that if $P$ is a polygonal environment, $G_{D i r}$ is a strongly connected direction constraint graph and $R$ is a piecewise linear path between start and end points $(s, e)$ then $R$ can be approximated by a $G_{D i r^{-}}$admissible path. We first need to adjust Algorithm 5 to generate a padded tubular neighborhood. Function PaddedTubularNeighborhood accepts the polynomial environment $P$, a line segment $\left(r_{i}, r_{i+1}\right)$ and a padding factor $1 / 2^{k}$ and calculates a padded tubular neighborhood of $\left(r_{i}, r_{i+1}\right)$ which has a padding less than or equal to the width of the neighborhood scaled by the padding factor.

In this version of the algorithm we will first calculate an orthogonal frame over $r_{i} r_{i+1}$ by calculating $\left(c_{i}, 0\right)=\left(r_{i+1}-r_{i}, 0\right) \times(0,0,1)$, where $\left(r_{i+1}-r_{i}, 0\right)$ is $r_{i+1}-r_{i}$ considered as a 3-dimensional vector and based on that we calculate $T$ which maps $r_{i+1}-r_{i}$ to $(1,0)$ and $c_{i}=\left(r_{i+1}-r_{i}, 0\right) \times(0,0,1)$ to $(1,0)$, when $r_{i}$ considered as the 
origin. We should note that

$$
\left\|\left(r_{i+1}-r_{i}, 0\right) \times(0,0,1)\right\|=\left\|r_{i+1}-r_{i}\right\| \sin (\pi / 2)=\left\|r_{i+1}-r_{i}\right\| .
$$

As a result $T$ can be written as $\left\|r_{i+1}-r_{i}\right\| I U$ where $U$ is a unitary rotation and $I$ the identity. Since both $U$ and $I$ are isometric, this means that if $(x, y)=T(p)$ then $x=d\left(r_{i}, \Pi_{r_{i} r_{i+1}}(p)\right) /\left\|r_{i+1}-r_{i}\right\|$ and $y=d\left(p, r_{i} r_{i+1}\right) /\left\|r_{i+1}-r_{i}\right\|$ where $\prod_{r_{i} r_{i+1}}(p)$ is the orthogonal projection of $p$ on $r_{i} r_{i+1}$.

After applying the transformation $T=\left(c_{i}^{\prime} c_{i}\right)^{-1} x-r_{i}$ to $P$, which involves translating the origin to $r_{i}$ and applying linear transformation $\left(c_{i}^{\prime} c_{i}\right)^{-1} x$, we find the maximum width $\lambda$ and a box $B_{\lambda^{\prime}}\left(r_{i} r_{i+1}\right)$ containing $r_{i} r_{i+1}$ exactly in the same manner as we did in Algorithm 5 where $\lambda^{\prime}=\lambda\left(1-2^{-k}\right)$. To calculate a padding we rotate $P$ by 90 degrees and apply function TubularNeighborhoodForUnit to the upper and lower bounding line segments of $B_{\lambda^{\prime}}\left(r_{i} r_{i+1}\right)$ as illustrated in Figure 3.3.1. 
Function PaddedTubularNeighborhood

Data: A polygon $P \in \mathbb{R}^{2}$, a line segment $\left(r_{i}, r_{i+1}\right)$ inside $P$, padding factor of the from $1 / 2^{k}$

Result: A padded tubular neighborhood of $\overline{01}$ with its closure inside $P$ distinguished by $R=\left(p_{h \text { Padding }}, p_{v \text { Padding }}\right.$, padding,$\left.\lambda\right)$

/* calculating an orthogonal frame along $r_{i} r_{i+1}$

*/

$c_{i}^{\prime} \leftarrow\left(r_{i+1}-r_{i}\right)$

$c_{i} \leftarrow$ normal vector to $c_{i}^{\prime}$;

/* finding a rotation that maps $c_{i}^{\prime}$ to $(1,0)$ (i.e. $r_{i} \overline{r_{i+1}}$ to $\mathrm{x}-$ axis) and $c_{i}$ to $(0,1)$

$T \leftarrow\left(c_{i}^{\prime} c_{i}\right)^{-1}$

start $\leftarrow(0,0)$;

end $\leftarrow T\left(r_{i+1}-r_{i}\right)=(0,1)$

$P_{T} \leftarrow$ apply transformation $T$ to $P$ with $r_{i}$ as origin ;

$\lambda \leftarrow$ Tubular NeighborhoodForUnit $\left(P_{T}\right.$, start, end $)$;

$P^{\prime} \leftarrow$ apply a $90^{\prime}$ rotation to $P_{T}$;

$l \leftarrow$ Tubular NeighborhoodForUnit $\left(P^{\prime},\left(-\lambda\left(1-\frac{1}{2^{k}}\right), 0\right),\left(\lambda\left(1-\frac{1}{2^{k}}\right), 0\right)\right)$;

$\hat{P} \leftarrow$ translate $P^{\prime}$ by $(0,-1)$;

$r \leftarrow$ Tubular NeighborhoodForUnit $\left(\hat{P},\left(-\lambda\left(1-\frac{1}{2^{k}}\right), 0\right),\left(\lambda\left(1-\frac{1}{2^{k}}\right), 0\right)\right)$;

sidePadding $\leftarrow \operatorname{Min}\{l, r\}$;

padding $\leftarrow \operatorname{Min}\left\{\right.$ sidePadding,$\left.\lambda / 2^{k}\right\}$;

paddedLeftTop $\leftarrow\left[\begin{array}{ll}c_{i}^{\prime} & c_{i}\end{array}\right]\left[\begin{array}{l}0 \\ \lambda\end{array}\right]$;

leftTopCorner $\leftarrow\left[\begin{array}{ll}c_{i}^{\prime} & c_{i}\end{array}\right]\left[\begin{array}{c}0 \\ \lambda\left(1-1 / 2^{k}\right)\end{array}\right]$;

rightpPad $\leftarrow\left[\begin{array}{ll}c_{i}^{\prime} & c_{i}\end{array}\right]\left[\begin{array}{c}\text { padding } \\ 0\end{array}\right]$; 


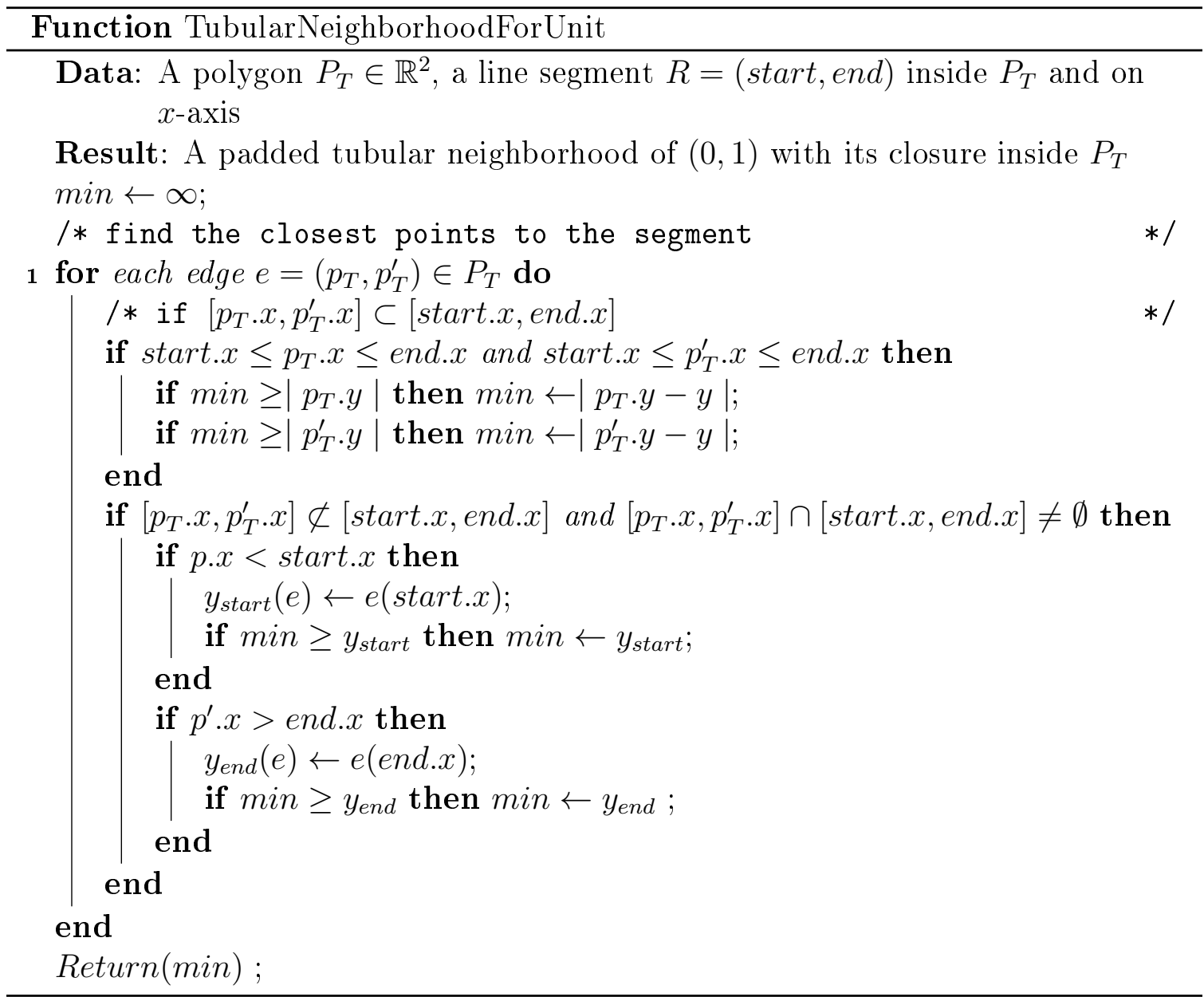

Theorem 26. Let $P$ be a polygonal environment (possibly with holes), $G_{\text {Dir }}$ strongly connected, spanning, and symmetric, $(s, e)$ a pair of points in $P$ and $R=(s=$ $\left.r_{1}, \cdots, r_{l}=e\right)$ a collision free piecewise linear path in $P$ between $s$ and $e$. Then for

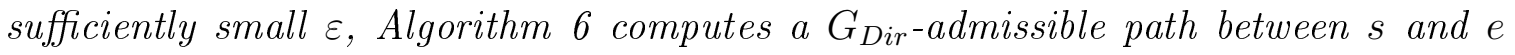
which is inside $P$.

Proof: Let $\Delta_{\text {Max }}$ be the maximum angular difference between two consecutive direction $v_{j}$ and $v_{j+1}$ in $V\left(G_{D i r}\right)$ ordered by $\leq_{\alpha}$. To calculate a $G_{D i r}$-admissible approximation of $R$ we will perform the following steps for each line segment $r_{i} r_{i+1}$ of $R$. 


\section{Algorithm 6.}

1. First we pick a $k$ to be used as a padding factor. We can easily choose $k=1$ for a padding factor of $1 / 2$. Larger $k$ also can be selected to enforce some grade of accuracy for $\varepsilon$.

2. We find a padded tubular neighborhood by running PaddedTubularNeighborhood on input $P, 2^{-k}$ and $r_{i} r_{i+1}$, and get $\operatorname{Box}_{\delta}\left(r_{i} r_{i+1}, \lambda\right)$ with padding $\delta \leq 1 / 2^{k} \lambda$.

3. Choose $\varepsilon<\min \left\{\delta, \cot \left(\Delta_{M a x} / 2\right) \frac{\lambda^{2}}{1+\lambda}\left\|r_{i}-r_{i+1}\right\|\right\}$ and $\beta=\varepsilon / \operatorname{Diam}\left(G_{\text {Dir }}\right)$. Since $d_{\max }(x, y) \leq\|x-y\|$ for any $(x, y)$ we can use $d_{\max }$ here to have computationally feasible option. We will discuss this step in more detail.

4. Calculate an approximation of $r_{i} r_{i+1}$ in $\operatorname{Box}_{\delta}\left(r_{i} r_{i+1}, \lambda\right)$ using $\varepsilon, \beta,\left(r_{i}, r_{i+1}\right)$ as start and end points and $\left(v_{s}(i), v_{e}(i)\right)$ as start and end directions. If we are processing $r_{1}=s$ we set $v_{s}(1)=v_{s}$ as start direction otherwise $v_{s}(i)=v_{e}(i-1)$. If we are processing the last leg of $R, r_{l-1} r_{l}$, we will set $v_{e}(l)=v_{e}$ otherwise $v_{e}(i)=v_{2}(i-1)$.

To be more clear on Step 3 it should be noted that the transformation used by PaddedTubularNeighborhood is transforming the orthogonal frame $\left\{c^{\prime}=r_{i+1}-r_{i}, c\right\}$ to $(\{(1,0),(0,1)\})$. As a result the values acquired by this algorithm for $\lambda$ and $\delta$ are scaled by $\left\|r_{i+1}-r_{i}\right\|$. In other word the distance between $r_{i}$ and the left top corner of the tubular neighborhood $\lambda^{\prime}$ is equal to $\lambda\left\|r_{i}-r_{i+1}\right\|$. Based on Lemma 24, $\varepsilon$ should be selected in a way that $\varepsilon<\delta$ and $\lambda^{\prime} \cot \left(\Delta_{\text {Max }} / 2\right)-\varepsilon\left(\left\|r_{i+1}-r_{i}\right\| / \lambda^{\prime}+1\right)>0$. By replacing $\lambda^{\prime}$ (the width of the tubular neighborhood) with $\lambda\left\|r_{i}-r_{i+1}\right\|$, the inequality in (3.3.1) converts to $\varepsilon<\cot \left(\Delta_{M a x} / 2\right) \frac{\lambda^{2}}{1+\lambda}\left\|r_{i}-r_{i+1}\right\|$ and since $d_{\max }(x, y) \leq\|x-y\|$ any

$$
\varepsilon<\cot \left(\Delta_{M a x} / 2\right) \frac{\lambda^{2}}{1+\lambda} d_{M a x}\left(r_{i}, r_{i+1}\right)
$$


will also work. We have $\cot \left(\Delta_{\text {Max }} / 2\right)=\frac{1+\cos \left(\Delta_{\text {Max }}\right)}{\sin \left(\Delta_{\text {Max }}\right)}$. Since $v_{j} \cdot v_{j+1}=$ $\left\|v_{j}\right\|\left\|v_{j+1}\right\| \cos \left(\Delta_{\text {Max }}\right)$ and

$$
\mathbf{c}=(0,0,1) \cdot v_{j} \times v_{j+1}=\left\|v_{j}\right\|\left\|v_{j+1}\right\| \sin \left(\Delta_{M a x}\right),
$$

we have

$$
\kappa:=\frac{\left\|v_{j}\right\|_{M a x}\left\|v_{j+1}\right\|_{M a x}+v_{j} \cdot v_{j+1}}{\mathbf{c}} \leq \cot \left(\Delta_{M a x} / 2\right)
$$

As a result condition (3.3.1) can be further simplified to

$$
\varepsilon<\kappa \frac{\lambda^{2}}{1+\lambda} d_{M a x}\left(r_{i}, r_{i+1}\right)
$$

Now we choose a $k^{\prime}$ such that $\varepsilon=d_{\max }\left(r_{i+1}, r_{i}\right) / 2^{k^{\prime}}$ satisfies

$$
d_{\max }\left(r_{i+1}, r_{i}\right) / 2^{k^{\prime}}<\kappa \frac{\lambda^{2}}{1+\lambda} d_{M a x}\left(r_{i}, r_{i+1}\right)
$$

or in other words $1 / 2^{k^{\prime}}<\kappa \frac{\lambda^{2}}{1+\lambda}$. The integer $k^{\prime}$ can be selected so large that the number of iterations in Step 3 stays the same as Algorithm Zigzag.

After selecting a suitable $\varepsilon$ we will choose $\beta<\varepsilon / \operatorname{Diameter}\left(G_{\text {Dir }}\right)$. The diameter of $G_{D i r}$ can be calculated using any all pairs shortest path algorithm but any upper bound such as the sum of the lengths of all edges in $G_{\text {Dir }}$ will also work. The above discussion shows that Step 3 can be done in constant time.

Step 1 is also done in constant time. Step 2 can be done in $O(n)$ for each linear piece of $R$. Step 4 can be computed in at most $\operatorname{Dimeter}\left(G_{D i r}\right) .\|s-e\| / \lambda \cot \left(\angle v_{i_{0}+1}-\right.$ $\left.v_{i_{0}}\right)$ iterations. As a result the whole process can be done in

$$
O\left(l\left(n+\operatorname{Dimeter}\left(G_{D i r}\right) \cdot\left\|r_{i}-r_{i+1}\right\| / \lambda \cot \left(\angle v_{i_{0}+1}-v_{i_{0}}\right)\right)\right)
$$

time. 
In this section, we have shown that for any polygonal environment $P$ (possibly with holes) and any symmetric, spanning, and strongly connected direction graph $G_{\text {Dir }}$, any piecewise linear map between two points $(s, e)$ can be converted to a $G_{D i r^{-}}$ admissible path. Since a piecewise linear map between two points in a polygonal environment can efficiently be found using classical methods like the one in [15], we can conclude that Problem 8 can be solved in two dimension.

If a pair of points $(s, e)$ is given in a polygonal environment $P$, then a piecewise linear map between $(s, e)$ can be found by traversing the graph built by connecting the center of the adjacent cells of the cylindrical algebraic decomposition of $P$ as outlined in [15]. The environment can be preprocessed in $O(n \log (n))$ expected time using the trapezoid method and then for any pair of query points $(s, e)$ a piecewise linear path $R$ can be reported in $O(n)$ time where $n$ is the size of $P$. Using the method in Theorem 26 this path can be approximated by a $G_{D i r}$-admissible path. 


\section{Chapter 4}

\section{Future Works}

\subsection{Approximation in higher dimensions}

We studied the problem of approximating an existing piecewise linear path between two points in a polygonal environment in 2 dimensions but most of the practical control problems involve more than 2 dimensions. This makes any generalization of the materials discussed in this thesis to higher dimensions very important. Unfortunately, calculating an obstacle avoiding piecewise linear path between two points is not very efficient in higher dimensions. This is because a cylindrical algebraic decomposition of a polytope may result in many cells. As shown in [6] and [17] the cylindrical algebraic decomposition of a dimensional polytope of size $n$ can generate $O\left(n^{2^{d}-1}\right)$ cells. This number can be improved to $O\left(n^{2 d-1}\right)$ using techniques in [18].

Before we go further let us exactly define what do we mean by polyhedral environment:

Definition 27. Let $A \subset \mathbb{Z}\left[x_{1}, \cdots, x_{d}\right]$ and $S=\left\{s_{1}, \cdots, s_{l}\right\}$ a decomposition of $\mathbb{R}^{d}$. We say $S$ is $A$ sign invariant ( $A$-invariant for brevity) if for each $f \in A$, the sign of $f(x)$ does not change for $x \in s_{i}$. S is called algebraic if each cell is a semi-algebraic set 
Definition 28. A set $S \subseteq \mathbb{R}^{d}$ is called semi-algebraic if it satisfies one of the following properties:

1. $S$ can be constructed by finite union, intersection, and completion of sets

$$
\left\{x \in \mathbb{R}^{d}, F(x) \geq 0\right\}
$$

where $F(x) \in \mathbb{Z}\left[x_{1}, \cdots, x_{d}\right]$ the ring of polynomial in $d$ variable with integer coefficients.

2. is a definable set with respect to theory of real closed fields with a quantifier free formula

A quantifier free formula is well formed formula of the theory of real closed fields which does not have any existential or general quantifier and a well formed formula is defined recursively by the following rules:

1. The formulas $a>b, a=b$ and $a<b$, for polynomials $a$ and $b$ in $\mathbb{Z}\left[x_{1}, \cdots, x_{d}\right]$ are well-formed.

2. if $\varphi(x)$ is a well-formed formula with free variable $x$ then so are $(\exists x ; \varphi(x))$ and $(\forall x ; \varphi(x))$.

3. if $\varphi$ and $\phi$ are well-formed formulas then so are $\varphi \wedge \phi, \varphi \vee \phi$ and $\neg \varphi$

A set $D$ in $\mathbb{R}^{d}$ is definable if there exist a well-formed formula $\varphi$ such that $D=$ $\left\{x \in \mathbb{R}^{d} ; \varphi(x)\right.$ is true $\}$ since by the quantifier elimination theorem we can eliminate quantifier for each well formed formula of the theory of real closed fields, the two properties in Definition 28 are equivalent.

Definition 29. A $d$ dimensional polyhedral environment is a semi-algebraic subset of $\mathbb{R}^{d}$ which is entirely defined by linear formulas (i.e., polynomials of degree 1 ). 
Most of the results discussed in previous chapters are based on $G_{D i r}$ being spanning and symmetric so the first step for generalizing our result to higher dimensions should involve a generalization of these properties to higher dimensions. Symmetricity of $G_{\text {Dir }}$ can be naturally generalized to higher dimensions as it does not assume any dependency on the dimension of the space. The generalization of the property of being spanning is not straightforward. Let us assume $G_{D i r}$ is a complete graph. We call $V\left(G_{\text {Dir }}\right) \subset T_{\overrightarrow{0}}\left(\mathbb{R}^{d}\right)$ spanning if for any set of directions $\left\{v_{1} \cdots v_{i-1}\right\}$ for any $i \leq d$ there exist a direction $v_{i}$ such that $\left\{v_{1} \cdots v_{i}\right\}$ is linearly independent. It is easy to observe that for $d=2$ this is equivalent to Definition 3. Now for a generic directional constraint graph $G_{D i r}$ we have:

Definition 30. Let $G_{D i r}$ be a direction constraint digraph and $V\left(G_{D i r}\right) \subset T_{\overrightarrow{0}}\left(\mathbb{R}^{d}\right)$ its direction set. $G_{D i r}$ is called spanning if for any path $\left(v_{1} \cdots v_{i-1}\right)$ of length $i-1$ for any $i \leq d$, there exists a $v_{i}$ in $\delta^{+}\left(v_{i-1}\right)$ such that $\left\{v_{1} \cdots v_{i}\right\}$ is linearly independent.

Based on this definition for being spanning, the higher dimensional version of Theorem 26 can be stated as follows:

Theorem 31. Let $P \subset \mathbb{R}^{d}$ be a polyhedral environment, $G_{\text {Dir }}$ strongly connected, spanning and symmetric, $(s, e)$ a pair of points in $P$ and $R=\left(s=r_{1}, \cdots, r_{l}=e\right)$ is a collision free piecewise linear path between s and e. Then for sufficiently small $\varepsilon$,

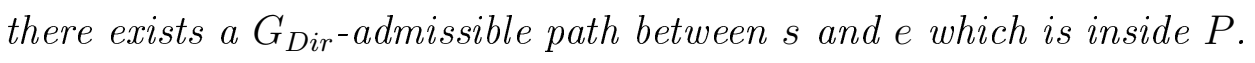

Let $s e$ be a line segment in $\mathbb{R}^{d}$ and $E=\left\{e_{1}, \cdots, e_{d}\right\}$ be an orthonormal base for $\mathbb{R}^{d}$ such that $s e \cdot e_{1}=0$, i.e., $e_{1}$ is in direction of $s e$. We define the tubular neighborhood along se and compatible with $E$ as

$$
\operatorname{Box}(E, \lambda, s e)\left\{x ; x=s+\alpha(e-s)+\sum_{i=2}^{n} \beta_{i} e_{i}, \alpha \leq 1, \beta_{i}<\lambda\right\} .
$$

Now let $P$ be a polytope in $\mathbb{R}^{n}$ and se a line segment inside $P$. Then for each selection 
of $E$, there exists a $\lambda_{E}$ such that $\operatorname{Box}(E, \lambda, s e) \subset P$.

The same techniques used in previous chapters seem to be generalizable to higher dimension, If the above definition for tubular neighborhood is used. The behavior and complexity of this generalization is yet to be studied.

\subsection{Direct calculation of $G_{D i r}$-admissible path and minimum turn}

We have mainly focused on approximating an existing piecewise linear path between two points by a $G_{D i r}$-admissible path. We also provided an algorithm to calculate a $G_{D i r^{-}}$admissible path directly in a triangulated polygonal environment when $G_{D i r}$ is a complete graph over the set $\{(1,0),(-1,0),(0,1),(0,-1)\}$. The same technique seems to be generalizable to higher dimensions for any polyhedral environment $P \subset \mathbb{R}^{d}$ and a simplical complex of $P$, when $G_{\text {Dir }}$ is the complete graph over the set of standard orthonormal bases of $\mathbb{R}^{d}$ plus their negatives. The exact behavior and complexity of this technique in higher dimensions is unknown.

We observed that the length of the $G_{\text {Dir }}$-admissible path generated by Algorithm 3 is related to how close the query points are to the vertices of the triangle. On contrary to classical versions of path planning problems this prevents us from giving a meaningful bound on the length of the $G_{D i r^{-}}$admissible path generated by Algorithm 4 in terms of the size of the environment. However the length of the $G_{D i r}$-admissible paths between the midpoint of edges of a triangle $T_{i}$ in triangulation of $P$ seems to be either one or two in most of the cases. This brings up the question of the expected length of a $G_{\text {Dir }}$ admissible path generated by Algorithm 4 .

The consequence of relaxing the constraints on $G_{D i r}$ to be only strongly connected, symmetric and spanning on Algorithm 4 is also the subject of future studies and not 
well understood.

Another interesting problem in this context is the problem of finding a $G_{D i r^{-}}$ admissible path between $(s, e)$ in such a way that the number of changes in directions are minimum. In this version of the problem we have a polygonal environment $P$ and a direction transition graph $G_{D i r}$ and for any start and end points $(s, e)$ we are interested in a $G_{D i r}$-admissible path between $s$ and $e$ inside $P$ which connect $s$ to $e$ with minimum number of turns. 


\section{List of References}

[1] L. E. Kavraki, P. Svestka, J.-C. Latombe, and M. Overmars. "Probabilistic roadmaps for path planning in high dimensional configuration spaces." IEEE Transactions on Robotics, 12 566-580 (1996).

[2] S. M. LaValle. "Rapidly-exploring random trees: A new tool for path planning." Technical report, Computer Science Department, Iowa State University (1998).

[3] A. M. Ladd and L. E. Kavraki. "Motion planning in presence of drift, underactuation and discerete system changes." In "Proceedings of Robotics: Science and System," (2005).

[4] D. Hsu, J.-C. Latombe, and R. Motwani. "Path planning in expansive configuration space." In "IEEE international Conference on Robotics and Automation, Volume 3, 2719-2726," (1997).

[5] J. F. Canny. Complexity of Robot Motion Planning. MIT Press (1988).

[6] J. T. Schwartz and M. Sharir. "On the piano movers problem ii: Topological properties of real algebraic manifolds." Technical report, Department of computer science Currunt Institute of Mathematical Sciences, New York University (1982).

[7] P. Cheng, G. Pappas, and V. Kumar. "Decidability of motion planning with differential constraints." In "Proceedings of IEEE International Conference on Robotics and Automation," (2007).

[8] F. Jean. "Complexity of nonholonomic motion planning." International Journal of Control, Volume 74, Issue 8 (2001).

[9] P. K. Agarwal, T. C. Biedl, S. Lazard, S. Robbins, S. Suri, and S. Whitesides. "Curvature-constrained shortest paths in a convex polygon (extended abstract)." In "Symposium on Computational Geometry," pages 392-401 (1998). 
[10] J.-D. Boissonnat and S. Lazard. "A polynomial-time algorithm for computing a shortest path of bounded curvature amidst moderate obstacles (extended abstract)." In "Symposium on Computational Geometry," pages 242-251 (1996).

[11] P. K. Agarwal, T. C. Biedl, S. Lazard, S. Robbins, S. Suri, and S. Whitesides. "Curvature-constrained shortest paths in a convex polygon." SIAM J. Comput. 31(6), 1814-1851 (2002).

[12] J.-D. Boissonnat, S. K. Ghosh, T. Kavitha, and S. Lazard. "An algorithm for computing a convex and simple path of bounded curvature in a simple polygon." Algorithmica 34(2), 109-156 (2002).

[13] J.-D. Boissonnat and S. Lazard. "A polynomial-time algorithm for computing shortest paths of bounded curvature amidstmoderate obstacles." Int. J. Comput. Geometry Appl. 13(3), 189-229 (2003).

[14] X. Goaoc, H.-S. Kim, and S. Lazard. "Bounded-curvature shortest paths through a sequence of points using convex optimization." SIAM J. Comput. 42(2), 662684 (2013).

[15] M. de Berg, O. Cheong, M. van Kreveld, and M. Overmars. Computational Geometry: Algorithms and Applications. Springer (2008).

[16] S. M. L. Valle. "Planning algorithms." Unknown Journal (2006).

[17] D. Arnon, G. E. Collins, and S. McCallum. "Cylindirical algebraic decomposition i: The basic algorithm." Technical report, Purdue University (1982).

[18] B. Chazelle, H. Edelsbrunner, L. J. Guibas, and M. Sharir. "A singly exponential stratification scheme for real semi-algebraic varieties and its applications." Theoretical Computer Science 84 (1991). 